\title{
L'islam au sud du Sahara. Une saison orientaliste en Afrique occidentale
}

Constitution d'un champ scientifique, héritages et transmissions

Islam South of the Sahara: An "Orientalist Season" in West Africa. Heritage and

Transmission in the Formation of a Scientific Field

Jean-Louis Triaud

\section{OpenEdition}

Journals

Édition électronique

URL : https://journals.openedition.org/etudesafricaines/16422

DOI : 10.4000/etudesafricaines. 16422

ISSN : 1777-5353

Éditeur

Éditions de l'EHESS

Édition imprimée

Date de publication : 20 novembre 2010

Pagination : 907-950

ISBN : 978-2-7132-2252-8

ISSN : 0008-0055

\section{Référence électronique}

Jean-Louis Triaud, "L'islam au sud du Sahara. Une saison orientaliste en Afrique occidentale », Cahiers d'études africaines [En ligne], 198-199-200 | 2010, mis en ligne le 02 janvier 2013, consulté le 21 avril 2022. URL : http://journals.openedition.org/etudesafricaines/16422 ; DOI : https://doi.org/10.4000/ etudesafricaines. 16422

Ce document a été généré automatiquement le 21 avril 2022.

(c) Cahiers d'Études africaines 


\title{
L'islam au sud du Sahara. Une saison orientaliste en Afrique occidentale
}

\author{
Constitution d'un champ scientifique, héritages et transmissions \\ Islam South of the Sahara: An "Orientalist Season" in West Africa. Heritage and \\ Transmission in the Formation of a Scientific Field
}

Jean-Louis Triaud

1 L'islam au sud du Sahara... Dans un temps pas si éloigné, on parlait volontiers d'« islam noir ", et cette dénomination elle-même est tout un programme. La formule voulait marquer les différences avec l'islam arabe et enclore l'islam subsaharien dans une chasse gardée. Le terme s'est banalisé. Les Éditions du Seuil jugèrent bon de donner ce titre à l'ouvrage de Vincent Monteil $(1964,1971,1980)$, qui n'en faisait pas cas. Dans la préface de la dernière édition, il justifiait ainsi le titre par défaut : «Je sais que le titre, L'Islam noir, prête le flanc à la critique et que des Africains musulmans me l'ont amicalement reproché. Mais, puisque, tel qu'il est, il aborde son troisième voyage, il m'a semblé qu'il valait mieux le garder, car, après tout, c'est sous ce nom qu'il s'est fait connaître. »

2 Puis, selon un processus connu, la conceptualisation coloniale fait l'objet de différentes formes de réappropriation. C'est que, de nos jours, cet islam subsaharien se prend à revendiquer sa négritude, à faire la jonction avec les revendications culturelles des fondateurs de la négritude (Seck 2008), non pour prêcher une dissidence ou un écart quelconque, comme l'auraient souhaité les acteurs coloniaux mais, au contraire, pour revendiquer une plus grande fidélité à l'esprit et à la lettre des textes classiques. L'idée d'un «islam noir", sans la formule, resurgit donc avec une nouvelle charge émotionnelle et une tout autre signification.

3 C'est ainsi que la marge dit sa fierté face à une certaine condescendance de la part du monde arabe (ibid.). Vieille marge et marche de la civilisation islamique, cet « islam noir » avec ou sans le nom, jadis provincial, multiplie ainsi les signes d'affirmation de soi, car son combat pour la reconnaissance de son espace culturel, d'ailleurs morcelé, vise aussi bien ce qu'il est convenu d'appeler l'Occident que l'Orient. 
4 Le chercheur, lui, ne peut que courir après un objet aux multiples costumes. Géographiquement d'abord, puisque l'islam subsaharien s'étend le long d'une large bande de terres, notamment soudano-sahéliennes, aux limites méridionales mobiles qui, pour faire simple, vont du Sénégal jusqu'au lac Tchad, et, dans un sens large, jusqu'en Somalie et Tanzanie. L'objet n'est donc pas homogène. Il apparaît comme une sorte de halo concentrique au-delà du monde arabo-musulman central. Cette géographie s'inscrit évidemment dans la longue durée de l'histoire.

5 Multiples costumes aussi d'un objet dont l'observation et l'analyse sont tout, sauf méthodologiquement homogènes. Chacun le revendique volontiers comme une dépendance évidente de sa propre spécialité : historiens, anthropologues, politologues notamment, géographes aussi. On omet parfois la dimension plus proprement orientaliste.

6 Nous voudrions, en effet, examiner ici le mode de constitution de ce champ scientifique sous l'angle particulier des travaux de traductions, d'éditions de textes et de catalogues de manuscrits. Nous prendrons l'espace francophone comme terrain d'observation, car, avant d'envisager une transversalité nécessaire avec l'espace anglophone, un retour sur les généalogies francophones, un réexamen des chaînes de transmissions nous paraît indispensable ${ }^{1}$. Les générations anglophones plus récentes vont croiser notre chemin et trouveront donc une place, le moment venu, dans notre démonstration.

\section{L'initiation faidherbienne}

7 À la charnière des derniers grands récits de voyage et des débuts de l'occupation coloniale, il y eut Faidherbe. Avant d'en venir à l'objet islamique, ou orientaliste, proprement dit, il convient de donner à cette figure la place qui lui revient. Place qui, nous allons le voir, n'est pas, pour notre sujet, aussi décisive qu'on pourrait l'imaginer, mais sur laquelle il est, en même temps, impossible de faire l'impasse.

8 Les «années Faidherbe" sont dominées par le modèle algérien (Pasquier 1974; Barrows 1977). Faidherbe est, en effet, le premier "Algérien » de marque, à travers lequel s'opère un transfert des expériences d'occupation coloniale acquises en Algérie. Faidherbe, officier d'artillerie et du génie, avait servi en Algérie pendant près de huit années, de 1842 à 1847, et, de nouveau, de 1849 à 1852. Entre-temps, il avait aussi été envoyé dans une colonie d'Ancien Régime, la Guadeloupe, en 1848-1849.

Il avait fait ses premières armes dans la province d'Oran, de 1844 à 1847, à l'époque de la lutte contre Abd El-Kader. Lors de son second séjour, il avait participé à une série d'opérations militaires de conquête: celles du général Camou dans le Djurdjura, du général Saint-Arnaud en Petite Kabylie, de sinistre mémoire, etc. Son expérience était donc celle des campagnes de conquête, et non des Bureaux arabes.

10 En 1852, il fut envoyé au Sénégal comme sous-directeur du génie. Il s'y distingua, à nouveau, par ses faits d'armes, et, le 16 décembre 1854, fut nommé gouverneur de l'embryon de colonie du Sénégal. Il exercera les fonctions de gouverneur du Sénégal à deux reprises, de 1854 à 1861, puis de 1863 à 1865.

11 Joël Glasman (2002) décrit son rôle d'initiateur scientifique à la tête de cette colonie naissante :

« Il arrive à ce poste avec l'ordre de conduire l'expansion territoriale décidée à

Paris et s'entoure rapidement d'une équipe avec laquelle il procède à la collecte systématique de données scientifiques [...]. Faidherbe met en place, pour faciliter le 
travail d'exploration et de rédaction, une politique de rassemblement de la documentation [...]. [Il] veille lui même au rassemblement des informations ${ }^{2}$ et accumule en personne de grosses masses documentaires. »

\section{excellence, qui est celui de Bonaparte en Égypte.}

«Plus que l'exemple algérien, c'est le modèle égyptien de Bonaparte et de l'Institut d'Égypte, animé par le bataillon des savants, qu'il faut invoquer ici ».(Saint-Martin 1989 : 272)

Tel est sans doute le meilleur éclairage que l'on puisse donner de cette aventure scientifique et de sa généalogie mentale. En cela, la période fécondante faidherbienne est une initiation à l'ethnologie et à la cartographie de la Sénégambie (Schmitz 1998 : 36). La phase proprement orientaliste va émerger plus tard. Les institutions de l'islam algérien en situation coloniale servent de ressources politiques à Faidherbe, mais les recherches sur l'islam sénégalais n'occupent pas une place centrale dans la production de l'équipe Faidherbe. Cependant, la confrontation avec Al-Hajj 'Umar crée une inquiétude durable au sujet de la confrérie tijânî. Mais ici encore, nous sommes plutôt dans l'ordre du politique. En d'autres termes, l'étude de l'islam et des textes islamiques n'est pas encore constituée en champ spécifique.

\section{La relégation de l'« islam noir»}

17 Ce champ scientifique, en effet, n'allait pas de soi. Il existait tout d'abord, au XIX siècle, une inégalité de traitement manifeste entre l'Afrique septentrionale et l'Afrique subsaharienne. Longtemps, l'étude de la première fut plus noble que la seconde. Les administrations et les corps militaires eux-mêmes épousaient et entretenaient cette distinction. Pour des raisons évidentes, la constitution d'un appareil scientifique sur l'islam commença en Algérie. L'Algérie et le Maghreb y étaient valorisés. L'islam 
subsaharien, dans ces terres inconnues et barbares, n'en était, au mieux, qu'une pâle copie. Cette mise en périphérie s'accordait bien avec la vision propre au monde arabe, qui regardait les pays subsahariens avec un complexe de supériorité, les considérant comme un ensemble de terroirs sans intérêt culturel, réserve d'or et d'esclaves, antre de «fétichistes ». Cette image dépréciative devait perdurer.

L'orientalisme épousa à son tour cette vision du monde, faite d'un centre évolué et d'une périphérie négligeable et sous-développée. On a assez reproché à l'orientalisme d'avoir conçu un objet dans lequel l'« Orient » dominé était lui-même en périphérie de l'œcoumène civilisé. L'islam subsaharien devint, lui-même, périphérie d'une périphérie. Tandis qu'il produisait des instruments érudits (dictionnaires, éditions critiques, inventaires de manuscrits) concernant les terres arabes centrales et d'autres pays musulmans d'Asie, l'orientalisme français du $\mathrm{xIX}^{\mathrm{e}}$ siècle et du début $\mathrm{du} \mathrm{xx}^{\mathrm{e}}$ ignora largement, à quelques exceptions signalées près, l'horizon subsaharien. Là n'étaient pas les matériaux nobles. Nous nuancerons donc le propos de Jean Schmitz (1998: 50) qui considère que c'est la construction de la théorie de l'« islam noir » qui aboutit «à une disqualification de la production littéraire arabe au sud du Sahara». Cette disqualification est beaucoup plus ancienne et elle est inhérente au dispositif orientaliste.

Il a donc manqué aux études sur l'islam subsaharien une "accumulation orientaliste " précoce. On admettra que la compréhension des phénomènes islamiques ne saurait se réduire à l'érudition textuelle. Du moins est-il réciproquement difficile d'y échapper.

Il y eut un autre clivage bien connu. Dans la constitution des disciplines, au XIX siècle, l'orientalisme (pour les sociétés pourvues d'une écriture) et l'ethnologie (pour les sociétés sans écriture) se partageaient l'étude des mondes jugés étrangers à l'historicité des sociétés industrielles.

21 L'islam subsaharien se trouva, dans ces conditions, à la lisière des deux disciplines, qui, toutes les deux, le rejetèrent. Les orientalistes, on l'a dit, s'intéressaient fort peu à ces musulmans de seconde zone. Ceux-ci furent dès lors assignés à un secteur de connaissance strictement utilitaire et appliqué: ce qu'on a appelé les «Affaires musulmanes ", c'est-à-dire un savoir fondé sur les seuls besoins de l'administration coloniale.

22 L'ethnologie de l'époque aboutissait à un déni parallèle. L'islam n'était, à ses yeux, qu'un corps étranger, une immixtion culturelle plutôt fâcheuse ${ }^{5}$. Dans ces conditions, l'étude de l'islam n'avait pas sa place dans les études africaines. Ce rejet par l'ethnologie, matrice principale (Sibeud 2002), quoique non unique, des études africaines en cours de constitution fut lourd de conséquences: c'était nier toute légitimité à l'objet. L'histoire documentée de ces rapports difficiles entre ethnologie et islam subsaharien reste à faire ${ }^{6}$. Elle éclairerait mieux ces quelques notations, nécessairement rapides.

23 L'« islam noir » s'est ainsi trouvé relégué dans le champ d'application des théories et stratégies administratives, entre les mains de spécialistes formés au Maghreb, les uns de passage, les autres à poste plus fixe. Ce furent principalement Robert Arnaud, Alfred Le Chatelier et Paul Marty, le premier administrateur civil, les deux autres officiers.

24 Arnaud, tourné vers la Mauritanie et soucieux d'instrumentaliser des personnages islamiques au service de la conquête, fut le premier responsable, à partir de la fin de 1905, à Dakar, du suivi des Affaires musulmanes ${ }^{7}$. Le Chatelier réalisa, lui, en 1887, une 
tournée d'enquête dans l'ouest de l'AOF. Son ouvrage, publié en 1899, fut la première somme de connaissances sur le sujet. Quant à Marty, premier responsable du Service des affaires musulmanes en 1912, il est l'auteur, d'une série encyclopédique (Marty 1913-1930: neuf titres), dont la matière était tirée directement des rapports administratifs venus de toute l'AOF. Tous étaient arabisants, mais aucun ne s'inscrivait dans les disciplines académiques. L'islam subsaharien relevait, dès lors, d'un genre particulier, celui d'une expertise administrative, vite répétitive, et formant un corpus où les classifications abusives et les présupposés aberrants voisinaient avec de véritables filons (biographiques, textuels, ou analytiques).

\section{L'expertise des Affaires musulmanes} subsaharien, ainsi placé dans cet " entre-deux ", fut livré dès lors à tous les fantasmes coloniaux sur le péril islamique, les complots confrériques, le féodalisme des marabouts, etc. Que ces fantasmes fussent, à l'occasion, confirmés par quelques affaires concrètes n'enlève rien à la caractérisation que l'on peut faire d'une représentation d'ensemble, qui, elle, fut constamment imprégnée d'une vision policière de l'islam ${ }^{8}$. On mesure alors mieux l'exceptionnelle richesse et ambiguïté de pareils corpus constitués par la puissance occupante, et tenant lieu, en quelque sorte par défaut, de science sur le sujet.

Le lexique de cette littérature mériterait d'être analysé comme tel. C'est le vocabulaire et le répertoire de toutes les théories du complot, avec les rumeurs érigées en certitudes, les collusions supputées avec l'étranger, les stratégies secrètes prêtées aux acteurs. Nous avons montré comment ce mécanisme a culminé autour de la confrérie Sanûsiyya jusqu'à devenir un modèle du genre (Triaud 1995). À certains égards, la dénonciation du hamallisme empruntera le même schéma dans l'espace ouest-africain, dans les années 1930 et 1940 (Savadogo 1998). Ce savoir a laissé des traces, notamment dans la littérature publiée entre les deux guerres et dans les rapports d'archives. Ces documents nous en disent souvent beaucoup plus sur les hantises et la grille d'analyse des observateurs que sur les musulmans "concrets" eux-mêmes. Il représente cependant une véritable tradition qu'il convient d'apprécier comme telle.

Cette tradition se perpétue au fil des rapports et trouve son conservatoire privilégié dans le СНEAM $^{9}$, dont la documentation inégale juxtapose des mémoires de stages et d'expériences de terrain, originaux ou compilateurs, parmi lesquels on trouve certains documents de valeur. Cette tradition-là trouve son point d'orgue dans l'ouvrage publié par Jean-Claude Froelich (1962), alors directeur du CHEAM, sous le titre Musulmans d'Afrique noire. Un autre ouvrage qui se veut différent sinon critique et concurrent, est celui publié deux ans plus tard sous le titre L'Islam noir, par Vincent Monteil ${ }^{10}$, un homme issu, lui aussi, des mêmes filières des Affaires indigènes (au Maroc) et du renseignement (le BCRA, le service de renseignements de la France Libre). Ce livre marque, en réalité, une rupture intellectuelle avec cette tradition des «Affaires musulmanes " et en clôt la série. Il traite, en effet, du sujet avec une empathie inhabituelle et met fin à la culture de la condescendance et du soupçon qui prédominait auparavant. Il a parfois été de bon ton, dans le monde des chercheurs, d'en contester le caractère scientifique. Destiné à un large public plutôt qu'à des cercles spécialisés, l'ouvrage reste cependant une mine d'informations. C'est un ouvrage de transition, 
dont l'un des mérites principaux réside, précisément, dans ce renversement des perspectives. Différents articles scientifiques complètent, en outre, l'activité éditoriale de Monteil à ce moment-là ${ }^{11}$.

On ne saurait sous-estimer cette longue tradition d'expertise coloniale. Elle imprègne le terrain de la recherche, lui sert parfois de bibliothèque de référence à défaut d'une véritable bibliothèque orientaliste. Elle a pu aussi servir de repoussoir, en rendant l'objet «islam» suspect, trop fortement connoté par le discours des Affaires musulmanes (Triaud 1997). Cette littérature de surveillance a, en outre, contribué à pérenniser l'idée que la « question islamique » relevait décidément d'autres paradigmes que ceux des "Études africaines». Dans tous les cas de figure, c'est une assise de savoirs dont l'évaluation exacte est toujours un préalable nécessaire.

\section{Une saison orientaliste}

29 À côté de cette longue filiation d'experts de la surveillance qui accumulent des « savoirs appliqués », il convient de faire maintenant place à une catégorie différente, qu'on aurait tort de confondre avec la précédente, celle des administrateurs-savants. L'intérêt de ces administrateurs est principalement intellectuel et culturel, et souvent distinct des besoins politiques immédiats, quand bien même les contacts humains qu'ils impliquent sont utiles à l'exercice de leur administration.

À vrai dire, les autorités considèrent le plus souvent ces recherches comme des signes d'originalité. "Il se consacra à ses chers travaux de linguistique ", disaient de Henri Gaden ses confrères à sa mort (Pondopoulo 2002 : 31). A. Pondopoulo (ibid. : 30) relève un propos de H. Gaden, dans une préface à l'ouvrage de Gilbert Vieillard, Notes sur les coutumes des Peuls du Fouta Djallon, où il «évoque la solitude du fonctionnaire colonial qui "s'intéresse aux populations qu'il dirige", et dont la curiosité reste étrangère à ses confrères ».

31 Trois noms s'imposent dans cette catégorie, ceux d'Henri Gaden et de Maurice Delafosse, auxquels il convient d'associer celui d'Octave Houdas, bien qu'il ne fût pas administrateur. C'est sous leur égide que s'ouvre une époque orientaliste, caractérisée par le souci d'éditions de textes et de constitution de dictionnaires.

32 Maurice Delafosse (1870-1926) ${ }^{12}$ est un des pères fondateurs des « Études africaines » en France. Se faisant linguiste, ethnologue et historien, il a posé les jalons de toute une série de problématiques. Mais c'est ici le traducteur qui nous intéresse. Très tôt, un partenariat savant s'est mis en place entre son professeur et beau-père arabisant, Octave Houdas, et lui. Louise Delafosse (1976: 293), fille de Maurice et petite-fille de Houdas, écrit que son grand-père « depuis longtemps, s'intéressait au Soudan autant qu'à l'Afrique ». Disposition rare chez un arabisant d'Alger.

33 Orientaliste reconnu, Houdas fit carrière à Alger (enseignant au lycée, puis à l'École des Lettres), avant de devenir en 1884, "professeur d'arabe vulgaire à l'École des Langues orientales vivantes de Paris» (Triaud 1998b : 213-214, 228). Il n'enseignait pas l'arabe classique ou littéral, la matière noble, mais l'arabe dialectal maghrébin, dont le but était précisément de former des praticiens de la gestion coloniale. Cependant, Houdas n'était pas étranger à l'activité intellectuelle de l'« École d'Alger ». Il publia, en 1903, avec William Marçais, un des grands noms de cette École, l'un des deux principaux recueils de hadith- celui d'al-Bukhârî, activité à la fois « monumentale » et orientaliste s'il en est (Houdas \& Marçais 1903). 
, Houdas fut aussi le premier à traduire et éditer en français, en 1898-1900, l'une des sources majeures de l'histoire du Soudan occidental, un texte en arabe écrit par un érudit du XVII ${ }^{\mathrm{e}}$ siècle de la boucle du Niger, le Ta'rîkh al-Sûdân (Es-Sa'di 1898-1900). Les exemplaires du manuscrit avaient été communiqués à Houdas par Archinard, Félix Dubois et le $\mathrm{D}^{\mathrm{r}}$ Tautain (Houdas 1898-1900 : préf.). Delafosse n'était pas encore de la partie et c'est le nom d'un autre diplômé des Langues orientales, Edmond Benoist, qui figure comme collaborateur de l'édition. Mais Delafosse, qui n'a encore jamais mis les pieds en Afrique subsaharienne, est déjà dans le sillage de Houdas. À l'âge de vingt ans, en 1890, il a commencé des études d'arabe à l'École des Langues orientales et obtenu, en 1895, le diplôme de l'« École spéciale des Langues orientales vivantes » (Delafosse 1913) " en langue arabe vulgaire» (Triaud 1998b : 214). Un tel diplôme, qui sera suivi d'un séjour de dix ans en Côte-d'Ivoire, donc loin du monde arabophone, ne faisait pas de lui un arabisant performant. Il lui en resta une initiation sérieuse qui, jointe au compagnonnage intellectuel avec Octave Houdas, dont il épouse, en bon talibé, la fille en 1907, l'introduit à un intérêt particulier pour la traduction. Le grand œuvre des deux hommes est la traduction et l'édition de l'autre chronique soudanaise majeure, le Ta'rîkh al-Fattash (Ka'ti 1913). C'est dans une lettre de 1912 à Charles Monteil ${ }^{13}$ que des informations importantes apparaissent à ce sujet :

«M. Terrier ${ }^{14}$ avait accepté [...] une traduction que j'ai faite de chroniques arabes relatives aux Ouagadou, aux Diawara, aux Diawambé, aux Massassi, à El-hadj-Omar, et cette traduction qui devrait paraître en juin et juillet n'a pas passé encore (Delafosse 1913) [...]. Je traduis en ce moment une chronique arabe du Fouta Toro, recueillie par Gaden ${ }^{15}$, qui est assez intéressante ; elle sera publiée dans la collection de la Revue du Monde musulman. Mon beau-père, qui est ici avec nous, traduit un manuscrit tout à fait curieux rapporté de Tombouctou par Bonnel de Mézières et qui complètera de façon très heureuse le Tarikh es Sudân, auquel il est légèrement postérieur $»^{16}$.

Sur le niveau exact de compétence en arabe de Maurice Delafosse, on trouve une réponse précise dans sa correspondance avec Gaden, à propos des Chroniques du Foûta : «Si j'étais capable de faire la besogne, je m'y mettrais bien, mais je suis par trop incompétent en arabe : il me faut un dictionnaire pour un mot sur six ou sept, ce qui cause une perte de temps énorme, sans compter les contre-sens possibles $! »^{17}$.

Houdas recevait, de son côté, le concours de Delafosse pour la transcription des noms propres et des termes soudanais. "L'équipe fonctionnait comme une alliance entre un "africaniste" et un "orientaliste" " (Pondopoulo 2004: 153). Le partage des tâches apparaît ainsi clairement entre le maître et le disciple. C'est Houdas qui choisit les textes ${ }^{18}$ et dirige l'atelier de traduction.

Jean Schmitz (1998 : 108 ; Schmitz dans Schmitz \& Bousbina 1998) s'est intéressé à cette phase orientaliste ${ }^{19}$. "La biographie de Delafosse, écrit-il, témoigne d'une sorte d'oscillation entre l'orientalisme et l'ethnographie [...]. » Il ajoute, et nous y adhérons, que «l'intérêt pour les langues africaines était médiatisé paradoxalement par sa connaissance de l'arabe, et donc par le paradigme lettré de l'orientalisme » (ibid.: 113). C'est sous cet éclairage et, ajouterons-nous, en suivant les modèles de « Langues O' » et d'Octave Houdas ${ }^{20}$, qu'il convient de placer, en effet, son goût pour les manuels de langues et les lexiques qui naît dès $1900^{21}$ et culmine avec son maître ouvrage en la matière, le dictionnaire de langue mandingue, toujours précieux, sorte de point d'orgue de son œuvre (Delafosse 1929, 1955), et dont les deux volumes seront publiés dans la «Bibliothèque de l'École des langues orientales vivantes ». Là où nous nuancerions à 
nouveau le propos de Jean Schmitz, c'est dans la qualification qu'il donne de cet orientalisme qu'il voit, dans la lignée de Faidherbe, comme un des éléments d'un vaste transfert de «techniques érudites» de l'Algérie vers l'AOF (Schmitz 1998 : 107-108). Qu'en est-il donc de l'influence de l'Algérie dans l'œuvre de Delafosse ?

Celui-ci est resté environ un an à Biskra en 1891-1892, d'abord dans cette curieuse «ONG » anti-esclavagiste de l'époque, les "Frères armés du Sahara » du cardinal Lavigerie, puis, au titre du service militaire, dans la garnison locale (L. Delafosse 1976 : 82-92). Même si ce séjour algérien, d'ailleurs pénible pour lui, l'initie à "l'Afrique ", il ne représente pas vraiment une propédeutique à l'étude du Maghreb, ni une familiarité avec les Bureaux arabes. Rappelons que Delafosse a quitté les Frères armés, malade et en désaccord implicite avec l'institution, qu'il a rejoint les zouaves, à Biskra, dans un mauvais état physique (ibid.). C'est d'ailleurs là que, à partir de son intérêt pour la lutte anti-esclavagiste, Delafosse se met au hausa, l'une des langues «transsahariennes » utiles, dont il a trouvé des locuteurs sur place, et prépare son lexique. Son intérêt le porte donc déjà vers le Sud.

Dans un article riche et documenté sur "Les Bureaux arabes et Maurice Delafosse ", Jacques Frémeaux (1998: 203) décrit le fonctionnement des Bureaux arabes et s'efforce d'ébaucher quelques liens en pointillés à propos du parcours de Delafosse: "Son expérience de jeunesse l'a mené directement chez les Pères Blancs de Biskra, en plein territoire militaire, où il serait bien étonnant qu'il n'ait pas eu quelques relations avec les officiers. » Il souligne ensuite les multiples relations que Delafosse a eues avec des officiers, plus tard, en Côte-d'Ivoire, comme commandant de cercle, ou comme administrateur chargé de la délimitation des frontières de la Côte-d'Ivoire. Delafosse, dont les ouvrages figuraient dans les bibliothèques de garnison, partageait avec les militaires, ajoute-t-il, une même adhésion à l'œuvre impériale et un sens de l'intérêt pratique de l'histoire coloniale et africaine. "Ces considérations à la fois politiques et patriotiques le rattachent très étroitement à tout le courant historique né au sein des Bureaux arabes un peu moins d'un siècle plus tôt $»^{22}$ (ibid. : 204). Jean Schmitz (1998: 107) affirme, dans le même sens, que «si Delafosse peut être considéré comme un des pères fondateurs de l'africanisme, c'est qu'il est également le dernier représentant de cette tradition des Bureaux arabes qui essaima vers les rives du Sénégal grâce à Faidherbe ».

L'hypothèse est séduisante, mais les indices insuffisants. Concrètement, Delafosse n'a pas eu l'expérience des Bureaux arabes. L'y rattacher peu ou prou relève d'une hypothèse commode mais non démontrée (si ce n'est l'affirmation putative de J. Frémeaux). Qu'il y ait des analogies entre sa pratique et celle des Bureaux arabes n'en fait pas un représentant, ni un élève de ceux-ci ${ }^{23}$. Il détestait la politique du sabre, privilégiait les rapports humains et visait à la connaissance des peuples soumis, mais on peut expliquer, autrement que par un modèle diffusionniste, ces affinités à distance. Le choix idéaliste initial de Delafosse s'est porté sur les «Frères armés du Sahara » du cardinal Lavigerie, dont les Bureaux arabes ne goûtaient guère l'immixtion dans leurs champs de compétence. Delafosse subit ensuite une expérience de garnison peu gratifiante et s'intéressa alors aux Hausa qui, eux, intéressaient beaucoup moins les Bureaux arabes.

41 En revanche, là où nous suivons volontiers la démonstration de Jacques Frémeaux (1998 : 204), c'est lorsqu'il écrit : "Mais Delafosse représente une autre tradition, plus spécifiquement savante. Il doit sa formation d'arabisant à Octave Houdas, qui fut son 
professeur à l'École des Langues orientales [...]. À partir de 1909, Delafosse enseigne luimême aux Langues orientales. »

Tous les chemins de Delafosse passent, en fait, par cette École, où il étudie, où il a son maître et futur beau-père, où il publie, et où il sera lui-même enseignant. Là est le modèle, là est la matrice. Le tandem Houdas-Delafosse s'inscrit dans cette logique. Comme le note Louise Delafosse (1976: 92), après l'épisode malheureux de Biskra, son père, dès son retour à Paris, en 1892, n'eut de cesse de revenir à l'École des Langues orientales et d'y retrouver son maître, et quand il s'absentera d'Afrique au cours de sa carrière, ce sera pour venir enseigner aux Langues $O$ '. Plus que d'une imprégnation algérienne, cet orientalisme-là est le produit des engagements personnels de deux hommes, Houdas et Delafosse, nourris de lexicographie et de philologie dans l'alma mater de la rue de Lille. Que Houdas ait commencé sa carrière à Alger, et qu'il y ait acquis son savoir et une part de sa notoriété, n'en fait pas exactement un relais des Bureaux arabes. Il s'agit donc, à notre sens, d'un attelage unique dans lequel les deux partenaires ont investi, par une délibération qui leur était propre, en poursuivant des intérêts neufs, les outils de la philologie orientaliste, celle même de Silvestre de Sacy (1758-1838), le père fondateur des études arabes et orientales en France qui, lui, ne devait rien à l'Algérie. Comme l'écrit précisément J. Schmitz (1998: 108), Delafosse "poursuit la quête et la traduction des textes arabes en vue d'inscrire l'Afrique dans une Grande Tradition [...] ». Là est le projet auquel Houdas et Delafosse collaborent par une passion personnelle partagée, appuyée aussi sur une idéologie commune fondée sur une conception plus « humaniste » de la colonisation. La préface écrite par Houdas (1898-1900 : IV, IX) à l'édition du Ta'rîkh al-Sûdân, avant même l'arrivée de Delafosse dans son entourage proche, illustre bien, à la fois les fondements de cette démarche et l'originalité du discours :

« [Cette Histoire du Soudan] montre que ces populations, auxquelles on est tenté de refuser toute initiative en matière de progrès, ont une civilisation propre qui ne leur avait pas été imposée par un peuple d'une autre race [...]. Enfin elle relie à l'histoire générale de l'humanité un groupe de nations qui jusqu'ici en avaient été complètement écartées.»

Si l'on ajoute dans le même passage une allusion quasi-métaphorique, quand bien même elle serait involontaire, à la conquête française: "La disparition de cet État relativement prospère [Songhay] est due en grande partie, sinon uniquement, à des conquérants de race blanche [Marocains]» (ibid. : IX), on est en présence d'un discours libéral, qui déborde celui des Bureaux arabes.

Il y a donc, entre les deux hommes, plus encore qu'un simple goût de l'érudition, une conviction commune concernant l'attitude à adopter à l'égard de la culture des populations conquises. Cet épisode orientaliste est si fortement lié aux deux hommes que la mort de Houdas (1916), puis celle de Delafosse (1926) y mettront fin: "Cet équilibre fragile entre ethnographie et orientalisme se brisera à la mort de Delafosse [...]. Il mourra sans avoir traduit deux œuvres importantes des confins de l'Occident musulman : le recueil des biographies de lettrés mauritaniens d'al-Baritayl [al-Bartili] ${ }^{24}$ et la monumentale histoire des États musulmans du Shaykh Muusa Kamara ${ }^{25}$ ", conclut à juste titre J. Schmitz (1998: 108). Cette saison orientaliste était donc plus la « petite " entreprise de deux chercheurs motivés, une heureuse conjonction, une singularité aussi, qui n'a pas eu d'héritiers ni de continuateurs, du moins immédiats. 

attelage. On a vu comment Delafosse et Gaden publient, en 1913, un manuscrit de Siré Abbas Soh sur l'histoire du Fouta, qui doit finalement beaucoup à Houdas. On rappellera aussi que Gaden avait fait recopier un exemplaire du Tedzkiret en-Nisiân, troisième grande chronique historique de la boucle du Niger pour la période d'occupation marocaine ( $\mathrm{xVI}^{\mathrm{e}}$-XVIII ${ }^{\mathrm{e}}$ siècles) et avait confié cette copie à Houdas. La traduction et le texte arabe furent édités par Houdas, toujours avec la collaboration de cet Edmond Benoist déjà cité, en 1899-190126. C'est dire que le processus de traduction de textes soudanais avait été initié par Houdas, à Langues $0^{\prime}$, avant même que n'apparaisse Delafosse. Gaden, un saint-cyrien, est de la même famille intellectuelle que Houdas et Delafosse, noms auxquels il convient d'ajouter celui de François Joseph Clozel (1860-1918), qui fut le protecteur du second tout au long de sa carrière ${ }^{27}$. Delafosse le suivit au Soudan ${ }^{28}$, puis à Dakar. C'est Clozel qui fonda, fin 1915, le Comité d'Études historiques et scientifiques de l'AoF, première institution scientifique " aofienne ${ }^{29}$. Il avait été élève de Houdas à l'École des Lettres d'Alger (Schmitz 1998: 113) et il était aussi diplômé d'arabe de l'École des Langues orientales, décidément au cœur de ce réseau intellectuel (Pondopoulo 2002:23). C'est précisément Clozel, alors gouverneur général, avec, à ses côtés, Delafosse, comme directeur des Affaires politiques de l' AOF, qui nomme Gaden en qualité de commandant militaire de la Mauritanie, en novembre 1916 (ibid.).

Gaden est de la même génération que Delafosse. Il est aussi de la même sensibilité : «Une ligne politique de dialogue avec l'islam, associée incontestablement avec l'étude des sociétés sahéliennes, de leurs langues et de leurs traditions écrites" (ibid. : 25). Delafosse est le père des études mandingues, Gaden, celui des études peules. Il y a, sur ce plan, un certain parallélisme entre eux: Gaden (1913-1914) publie, lui aussi, un grand dictionnaire de langue peule.

L'œuvre scientifique de Gaden est considérable ${ }^{30}$. Elle était menée en contact permanent avec un réseau d'interprètes et de lettrés (Pondopoulo 2002 : 27). Enfin, Gaden a été, avec Delafosse, au cœur d'une aventure érudite qui a dépassé ses moyens et ceux de ses collègues. Il a passé commande d'une "Histoire du Fouta " à un personnage religieux du Damga, Cheikh Moussa Kamara autour de 1923-1924. Kamara s'est pris au jeu, écrivant d'une plume prolifique une histoire monumentale de la région, collectant à cet effet documents écrits et oraux. Gaden et Delafosse souhaitèrent une traduction, et firent appel aux autorités en études arabes du moment, notamment Maurice Gaudefroy-Demombynes, lui aussi professeur à l'École des Langues orientales ${ }^{31}$. Boyer, le directeur de l'École des Langues orientales vivantes, était prêt à le publier dans la collection « Documents arabes relatifs à l'histoire du Soudan » de l'ELov, celle-là même où avaient été publiés les Ta'rîkh-s soudanais (Schmitz dir. 1998: 31) mais l'entreprise était trop lourde. Théodore Monod, directeur du nouvel IFAN, sollicité lui aussi en 1939, ne trouva pas davantage de solution. A. Pondopoulo (1993 : 95-110), qui rapporte en détail cette saga, montre comment toutes les autorités sollicitées « reculent devant l'ampleur et la cadence de la production littéraire de Kamara ». Mais, autour de Moussa Kamara - et l'homme est à cet égard un symbole ${ }^{32}-$ c'est tout $^{2}$ l'aréopage des grands noms des Affaires musulmanes et de l'orientalisme, qui, à un moment ou un autre, viennent se pencher sur sa personne et son entreprise.

J. Schmitz (1998: 113) caractérise en ces termes l'œuvre de Gaden: "Il était à la charnière de trois entreprises : l'annotation et l'édition de textes écrits en arabe sur 
l'histoire des Peuls [...], la traduction de textes en peul ou en ajami (en peul utilisant la graphie arabe), enfin l'histoire en français des différents royaumes wolofs par Yoro Dyao. Ces trois orientations de son activité donnaient lieu à des traductions dans trois langues : arabe, peul, français $~_{33}$. Faut-il y voir un «fil d'Ariane », comme il le pense, avec l'époque Faidherbe? Il nous semble plutôt qu'un «mythe des origines" s'est constitué autour de la période Faidherbe, qu'il conviendrait de nuancer au cas par cas. Nous croyons plutôt, dans ce cas, à l'existence d'une école orientaliste sui generis, composée de Houdas, Gaden et Delafosse, dont les filiations multiples passent, à certains moments, par l'Algérie et le Maroc, mais convergent surtout vers l'École des Langues orientales autour de projets qu'ils ont eux-mêmes conçus. Il y a une interaction manifeste entre ces trois figures dans ces travaux d'édition critique.

Cependant, dans cette architecture d'un moment orientaliste, il est nécessaire de faire une place, en quelque sorte latérale, à trois autres noms également importants, qui marquent l'orientalisme subsaharien de ce début du $\mathrm{xx}^{\mathrm{e}}$ siècle, et qui représentent, pour leur part, une intégration de l'espace maure dans la problématique des érudits spécialistes du Maghreb et du monde musulman central. Il s'agit de Massignon, Hamet et Basset.

Louis Massignon (1883-1962) ${ }^{34}$ ouvre la voie, avec l'inventaire de la bibliothèque des Ahl shaikh Sidiyya (1909), une grande famille savante du sud-ouest de la Mauritanie, celle fondée par Shaykh Sidiyya Al-Kabîr (1774-1868).

51 Cet intérêt de Massignon pour les manuscrits arabes encourage un effort de traductions. C'est dans ce cadre qu'on sollicite la contribution d'Ismaël Hamet, un interprète originaire d'Algérie ${ }^{35}$. On doit à celui-ci une série de traductions scientifiques de textes historiques maghrébins, et, pour ce qui concerne l'environnement sénégalais, la traduction de trois manuscrits arabes dans Chroniques de la Mauritanie sénégalaise (Hamet 1911) ${ }^{36}$. Après la Première Guerre mondiale, Ismaël Hamet sera chargé de faire le cours d'histoire du Maghreb (ibid. 1923) à l'Institut des hautes études marocaines, dont il deviendra plus tard le directeur.

René Basset (1855-1924), linguiste, spécialiste des langues arabe et berbère ${ }^{37}$, qui fut doyen de la Faculté des Lettres d'Alger, représente un autre exemple de relation privilégiée avec le Maghreb. On lui doit une «Mission au Sénégal», publiée dans le Bulletin de Correspondance africaine, qui était une publication de l'École des Lettres d'Alger. Il y étudiait notamment (Basset 1909, I) le parler berbère zénaga, prenant ici, effectivement, la suite d'un travail de Faidherbe (1877) sur le même parler, et, dans le même volume (Basset 1909, III), il utilisait largement l'œuvre en arabe d'un savant et poète daymani, M'Hammed Ould Ahmed Youra, sur l'histoire des puits de sa région ${ }^{38}$.

On observe à cette occasion comment se noue un compagnonnage scientifique entre Basset, Gaden et Marty. C'est le commandant Gaden, alors adjoint au commissaire du gouvernement général en Mauritanie, qui avait demandé au savant maure ce texte. C'est René Basset (1909, III) qui en fait un usage extensif dans Mission au Sénégal, et c'est Paul Marty, enfin, qui en donne la traduction en 1920. La chaîne est ainsi complète. Marty (1920: 311) ajoute que son travail «est offert aux lecteurs du Bulletin avec le double assentiment de MM. Basset et Gaden ».

54 Tous ces croisements mènent, on le voit, vers l'École, devenue Faculté, des Lettres d'Alger, ainsi qu'à l'Institut des hautes études marocaines. Il n'est pas tant question de Bureaux arabes que de filières d'érudition textuelle qui investissent progressivement la Mauritanie jusque dans ses confins sénégalais. Entre Houdas et Delafosse, tous les deux 
"Soudanais », et Massignon, Hamet et Basset ${ }^{39}$, plus tournés vers les sources maures, Gaden, qui fraye avec les uns et les autres, sert de lien. Dans tous les cas, le gros œuvre de ces traductions est terminé vers 1920.

\section{La marche vers l'autonomie : confréries, islam noir et maraboutisme}

Il nous faut maintenant mener une réflexion plus globale sur la relation de ces islamologues des textes, du terrain et de l'administration - souvent les trois réunis en une même personne - avec leurs aînés d'Afrique du Nord.

Le rapport avec les modèles orientalistes venus d'Algérie ou du Maroc est complexe. Il est fait d'emprunts initiaux, et aussi de ré-appropriations sur lesquelles on n'a pas assez insisté. Loin d'être une simple succursale du modèle algérien, le tandem HoudasDelafosse est innovateur. Il s'ancre au Sud et considère désormais le champ soudanais, non comme une périphérie, mais comme une province culturelle particulière avec sa propre centralité. La théorie de l'«islam noir» représente une autre de ces réappropriations: il s'agit précisément de séparer l'islam subsaharien de celui du Maghreb. Autonomiser l'islam subsaharien s'inscrit dans la logique de la constitution d'une entité «AOF " qui entend se différencier - aussi bien sur le plan de l'administration que sur celui des représentations - du premier modèle algérien.

Nous avons déjà souligné l'importance de la question confrérique dans la maturation de ce "séparatisme » (Triaud 1998a). L'intérêt pour les confréries, la fascination pour les confréries, vient des premiers temps de la conquête française en Algérie ${ }^{40}$. Cette catégorie participe de la taxinomie coloniale qui se met en place et devient un outil de classification et de recensement à l'égal des ethnies et des tribus. Elle s'intègre aussi dans les théories du complot, dans la peur française de l'islam ${ }^{41}$. Le cœur de l'islam serait dans ses confréries, organisations occultes toujours potentiellement subversives. La question confrérique va précisément être l'occasion d'une première rupture avec le modèle algérien.

Déjà, l'article de l'Interprète Marchand (1897), qui est une des premières monographies sur l'islam en Afrique de l'Ouest (Triaud 1998a : 274), part du modèle algérien à la recherche des confréries, mais c'est pour en conclure qu'il n'y a pas, au Soudan, de zâwiya « semblables à celles d'Algérie ".

Dix ans plus tôt, à la fin de l'année 1887, Alfred Le Chatelier était venu d'Algérie pour trouver, lui aussi, les confréries au sud du Sahara ${ }^{42}$.

Après avoir sacrifié une première fois au modèle confrérique à propos du Hedjaz (Le Chatelier 1887), ce saint-cyrien, officier des Affaires indigènes en Algérie (1876-1886) c'est-à-dire des Bureaux arabes (notamment à Ouargla, 1883-1885), dont il était un «pur produit» (Didier 1997 : 105), était parti en mission au Sénégal et au Soudan français à la fin de 1887, alors qu'il quittait les Affaires indigènes. Occupé par d'autres tâches, il mit du temps à publier son ouvrage (Le Chatelier 1899), destiné à devenir un premier classique de la bibliothèque coloniale. Sa réflexion, sans doute facilitée par la distance qu'il avait prise avec sa première carrière algérienne, avait mûri entre-temps. Il s'en explique très clairement dans sa préface : «Étudier sur les lieux, au Sénégal, au Niger, l'organisation des ordres religieux chez nos sujets nègres, tel devint l'objectif de mon voyage au Soudan, à la fin de 1887 » (ibid.: 6). Or son voyage met fortement à l'épreuve cette thèse, à une époque où les confréries sénégalaises n'avaient pas encore l'ampleur qu'elles ont prise depuis. Le Chatelier exprime le « regret » de s'être «trop 
exclusivement inspiré, dans des ouvrages antérieurs, de l'École algérienne » (ibid. : 10). Considérant que "l'École algérienne [...] s'est trompée", il conteste le schéma confrérique, celui d'associations toujours fortement structurées et de sociétés secrètes - un caractère, dit-il, «qu'elles n'ont pas toujours en Algérie et qu'elles n'ont qu'exceptionnellement ailleurs » (ibid. : 12). Parti pour chercher les confréries, avec le sentiment que, s'il ne les trouvait pas, "l'insuffisance de [ses] recherches devait être seule en cause » (ibid. : 13), Le Chatelier remet totalement en question ce paradigme. Le terrain subsaharien a ainsi servi de révélateur, inversant dès lors le sens du mouvement de la connaissance.

61 Le Chatelier fait ensuite une carrière brillante ${ }^{43}$. Il entre au Collège de France et crée la chaire de sociographie musulmane (1902), celle qu'occupera après lui Massignon. Il fonde la Mission scientifique du Maroc (1904) ${ }^{44}$, puis la Revue du Monde musulman (1906).

Delafosse s'inscrit parfaitement dans cette rupture, jusqu'à, finalement, la retourner contre Le Chatelier lui-même. En 1910, il se réjouit auprès de Gaden de sa récente association à la Revue du Monde musulman (Pondopoulo 2004: 257) ${ }^{45}$. Cependant, la protection de Le Chatelier devient vite pesante. Le directeur de la revue surévalue l'importance de l'islam pour les sociétés africaines dans lequel il n'est pas loin de voir un facteur central et civilisateur. Delafosse, en connaisseur du terrain, montre plutôt les limites de cet islam subsaharien, introduisant des distinctions utiles entre élites islamisées et populations restées proches du paganisme. Delafosse critique, chez Le Chatelier, "sa manie de ne vouloir laisser voir l'islam que sous des couleurs de rose", ajoutant qu'il ne voulait rien publier « qui puisse être considéré comme une critique de l'islam et des musulmans : c'est assommant » (ibid. : 258).

Delafosse expérimente alors un nouveau concept, celui de "maraboutisme» - un terme déjà utilisé dans la taxinomie administrative, qui parle aussi de "féodalités maraboutiques ", pour désigner des personnages religieux et leurs clientèles -, ces plus petites unités faisant, à ses yeux, sens dans le paysage, contrairement aux étiquettes confrériques. Devant la censure de Le Chatelier, Delafosse (1911) se rapproche alors d'Auguste Terrier, autre "Marocain $»^{46}$ et publie son article sur les confréries et le maraboutisme dans le Bulletin du Comité de l'Afrique française. Il y note très précisément que: «Nos habitudes de méthode et de classification, comme notre besoin de logique, nous conduisent trop facilement à unifier des choses qui n'ont souvent d'autre lien qu'une appellation commune » (Delafosse 1911 : 81-90). Il affirme, lui aussi, que les confréries ne représentent pas, au Soudan, la même réalité qu'au Maghreb, mais c'est pour introduire ce concept de maraboutisme, que récuse Le Chatelier.

Un processus d'autonomisation est donc en cours, qui débouche, en 1912, sur la théorie de l'« islam noir " ${ }^{47}$, formulée par Robert Arnaud (1912), autre "Algérien » d'origine, dans son ouvrage fondateur. C'est là qu'il appelle de ses vœux « un islam purement africain " et un "éthiopianisme musulman » (ibid. : 128-129). Pour le reste, Arnaud est d'accord avec Delafosse : ce ne sont pas les confréries, mais les marabouts qui peuvent constituer le véritable danger.

Arnaud, Delafosse et Clozel sont alors "au pouvoir ", dans ces années 1910, chacun dans son rôle propre, à Dakar. Ce sont ces autorités "aofiennes" qui incarnent le processus d'autonomie, c'est-à-dire la disjonction de l'islamologie pratiquée en AOF, de celle de l'Afrique du Nord. Il n'y a pas de contradiction, sur ce plan, entre le Delafosse 
orientaliste - d'un orientalisme appliqué au Soudan - et le Delafosse administrateur, engagé dans l'orientation de la politique musulmane au Soudan et au Sénégal.

Comme le souligne A. Pondopoulo (2004: 256-267) ${ }^{48}$ : «La polémique avec Le Chatelier [...] ne visait donc pas des questions purement académiques : elle évoquait une lutte d'influence et le désir de Delafosse d'établir les études africaines comme domaine indépendant, lequel, tout en profitant des traductions et des commentaires des orientalistes, tels ceux de René Basset ou Maurice Gaudefroy-Demombynes, ne se réduirait pas à une annexe de l'érudition philologique ou des études musulmanes [...]. Delafosse reprochait à Le Chatelier la volonté d'absorption des études africaines par celles de l'islam » (ibid. $2004: 261$ ).

En d'autres termes, même s'il ne conceptualise pas la chose avec les mêmes termes, Delafosse s'inscrit parfaitement dans la même logique que celle de l'« islam noir ». Les hommes des jeunes colonies du monde subsaharien entendent se doter de leurs propres instruments d'analyse, sans devoir en rendre compte à leurs " aînés » d'Algérie. C'est à la fois un processus d'autonomie coloniale et d'autonomie scientifique. Et cette « saison autonomiste ", loin d'être en rupture avec la "saison orientaliste » que nous avons décrite précédemment, en est comme le prolongement naturel. Elle repose sur des bases analogues ou convergentes : la revalorisation des sociétés subsahariennes comme terrain propre, l'analyse des textes comme outils d'« habilitation » d'une histoire et d'une ethnographie de ces sociétés, et l'adoption de concepts adaptés à ce terrain. La présence de Delafosse incarne bien cette continuité. On ne l'a pas assez remarqué : la théorie de l'« islam noir " vise, certes, à couper l'islam subsaharien des pays et des tuteurs arabes, mais elle vise tout autant à couper l'analyse de cet islam du monopole alors exercé par les tuteurs orientalistes, notamment ceux implantés au Maghreb, voire à Paris.

68 En dépit de ces mises en garde, la confrérie va continuer à fasciner les administrations qui y voient un outil de classification commode. Les chercheurs, à leur tour, privilégieront les confréries sénégalaises, qui ont prospéré en situation coloniale, et en feront un modèle dominant (Triaud 1998a). Ainsi, le «tout confrérique », certes venu d'Algérie, a-t-il traversé les générations et les disciplines jusqu'à incarner, à l'inverse, une image supposée emblématique de l'«islam noir»? C'est que, entre-temps, la confrérie mouride a surgi et prospéré ${ }^{49}$ comme pour donner raison à ceux que hantait la référence confrérique!

\section{Les héritiers de l'orientalisme soudanais}

Que reste-t-il de cette «vague » orientaliste que nous avons décrite entre Sénégal et Niger? Des œuvres fondatrices, mais pas de relève immédiate. Du moins, l'héritage culturel islamique subsaharien a-t-il conquis, grâce à elles, une première légitimité scientifique. Elles vont rester comme des balises, souvent citées et utilisées pendant la majeure partie du $\mathrm{xx}^{\mathrm{e}}$ siècle.

Après elles, il y a une longue coupure, et l'intérêt pour les textes ne reprend qu'avec le temps des indépendances ${ }^{50}$. On doit d'abord à V. Monteil (1965-1966), alors directeur de l'IFAN, un souci d'inventaire des manuscrits arabo-africains de l'IFAN, sous forme de «bilan provisoire ». Cet effort d'inventaire sera poursuivi par T. Diallo, M. B. M'Backé, M. Trifkovic et B. Barry (1966), E.-H. R. Mbaye et B. Mbaye (1975) et K. Mbacké et T. Ka (1994). 
71 On doit encore à A. Samb (1970, 1971, 1973, 1976), successeur de Monteil à l'IFAN, ainsi qu'à M. Ndiaye $(1976,1978)$, une reprise d'un effort de traduction, à partir d'éléments de l'œuvre de Moussa Kamara, dans les années $1970^{51}$.

Il convient, ensuite, de réserver une place particulière à plusieurs entreprises éditoriales qui, toutes, ont pour point commun le retour à la traduction et l'édition de textes.

73 Il y a d'abord la coopération établie, à partir de 1965 autour de l'association Classiques africains, pilotée par Éric de Dampierre et Claude Tardits, qui a pour but de «multiplier le nombre de documents littéraires sur lesquels pourront s'appuyer les recherches et les réflexions sur les civilisations de l'Afrique noire » (Kyburz 1994 : 483-488).

74 C'est dans cette collection que paraît, en 1965, Poésie peule de l'Adamawa, traduit et édité par P.-F. Lacroix, professeur de peul à l'École nationale des Langues orientales, que s'inscrivent ensuite les traductions du peul, par Alfa Ibrahima Sow (1966, 1968, 1971), de trois œuvres littéraires de Guinée ${ }^{52}$. A. I. Sow a longtemps enseigné le peul, lui aussi à l'École nationale des Langues orientales, et publié différents travaux (dictionnaires, recueils de textes, syllabaires) sur la langue et les parlers peuls.

75 Tout se passe comme si la langue peule, une des langues écrites de l'islam en Afrique de l'Ouest et au Cameroun, avait alors pris un moment l'avantage sur l'arabe dans le travail de traduction et d'édition savante. Du côté de l'aire mandé, les premières recherches sur le mandingue ou bambara en 'ajamî sont plus récentes (Tamari 1994, 2005).

76 Un second moment, important, notamment pour les historiens, est la publication de la traduction des sources arabes médiévales sur le Sahara et le monde subsaharien. Jusqu'alors, les chercheurs étaient tributaires d'une production orientaliste, superbe mais inaccessible ailleurs qu'en quelques bibliothèques nationales, les Monumenta Cartographica Africae et Aegypti (Kamal 1926-1951), édition de luxe en 16 volumes en grand in-folio, tirée à cent exemplaires seulement, et due au prince Youssouf Kamal et à une équipe de chercheurs internationaux, parmi lesquels le Français Charles de la Roncière, historien de la Marine. Les Monumenta recensaient toutes les sources alors connues, en différentes langues, sur l'Afrique, depuis l'époque pharaonique jusqu'aux découvertes portugaises, sans parler d'une large reproduction de cartes (Mauny dans Cuoq 1975 : XI-XII).

77 Le Recueil des sources arabes concernant l'Afrique occidentale du VIII au XVI siècle (Bilâd alSûdân) par Joseph Cuoq, commencée comme thèse de doctorat sous la direction de Raymond Mauny (Université Paris I) et préfacée par lui, fut publiée par l'Institut de Recherche et d'Histoire des Textes du CNRS (Cuoq 1975). Pour des raisons de coût, elle ne comportait pas de reproduction des textes arabes, mais elle offrait au public concerné, et notamment enseignant et étudiant, un corpus de soixante-douze auteurs ${ }^{53}$.

D'autres entreprises ont été menées, cette fois, sur les sources arabo-africaines. Il s'agit d'abord de la publication d'une étude bio-bibliographique sur Ahmad Bâbâ, cet intellectuel tombouctien emblématique, victime d'une longue déportation au Maroc. Ce travail, issu d'une thèse de doctorat de l'Université Paris IV, dirigée par Charles Pellat, un orientaliste étranger au monde subsaharien, était publié par un chercheur arabisant malien, Mahmoud Zouber (1977), fondateur et premier directeur du Centre de documentation et de recherches Ahmad Baba (CEDRAB), créé par l'UNESCO en 1970, et effectivement entré en fonctionnement en 1977. 
79 La même année, Dierk Lange publie le diwân des sultans du Kanem-Bornou (Lange 1977), une liste dynastique consignée en arabe, et couvrant plus de huit siècles. Ce travail de facture orientaliste, avec texte arabe, traduction en français, notes et commentaires, issu d'une thèse de doctorat soutenue à l'Université Paris I, offre au public scientifique une nouvelle source historique arabe sur un empire soudanais.

$\mathrm{Au}$ cours de la décennie suivante a lieu la publication, par Sidi Mohamed Mahibou et Jean-Louis Triaud (1983), du pamphlet polémique d'al-Hâjj 'Umar contre la Dina du Macina, Bayân mâ waqa'a, avec reproduction du texte arabe, traduction, notes et commentaires. Parallèlement à cette édition particulière, à partir d'un manuscrit arabo-africain de la Bibliothèque nationale de France - un de ceux qui composaient la bibliothèque d'al-Hâjj 'Umar et qui furent expédiés à Paris par Archinard après la conquête de Ségou (1890) -, une opération d'inventaire de l'ensemble du fonds fut conduite par David Robinson et Louis Brenner, à laquelle nous avons également participé. Le résultat fut la publication dans le même cadre (CNRS, IRHT) de l'Inventaire de la Bibliothèque 'umarienne de Ségou, qui avait été préparé par Noureddine Ghali et Sidi Mohamed Mahibou, deux arabisants, le premier tunisien, le second nigérien (Ghali, Mahibou \& Brenner 1985) $)^{54}$.

81 La publication de sources se poursuivait encore avec l'édition, par Michel Abitbol (1982), d'une chronique arabe traitant de Tombouctou au XvIII ${ }^{\mathrm{e}}$ siècle.

Dans le registre des inventaires de sources, il convient de réserver une place particulière à la Mauritanie, pays arabophone, qui fut un espace de production manuscrite intense. Plusieurs entreprises de catalogage se sont succédé à partir des années 1960. La première fut le catalogue provisoire établi par Adam Heymowski et Mokhtar Ould Hamidoun (1965-1966), qui recensait 2000 œuvres. Il fut tiré en quelques exemplaires, en arabe, avec transcription phonétique (Kane $2003: 9$ ).

83 Vingt années plus tard, Ulrich Rebstock publie le Katalog der arabischen Handschriften in Mauretanien, fruit de la collaboration entre une équipe de l'Université de Tübingen, et l'Institut mauritanien de la recherche scientifique. Ce catalogue (Rebstock et al. 1988) est entièrement en caractères latins et recense 2239 manuscrits dans le pays (Ould Cheikh 1987).

Le troisième travail, le plus complet, est le catalogue de manuscrits arabes de l'Institut mauritanien de recherche scientifique (IMRS) qui recense 3100 manuscrits collectés (Stewart 1990). A. W. Ould Cheikh, alors directeur de l'IMRS, joua un rôle majeur, avec Charles Stewart, dans cette entreprise. Ce dernier a, d'autre part, procédé à l'inventaire de la bibliothèque des Ahl al-shaikh Siddiyya à Boutilimit (Stewart 1994), avec dépôt conjoint du catalogue à Boutilimit et à Urbana-Champaign (États-Unis).

85 L'entreprise de publication suivante concerne Moussa Kamara ${ }^{55}$. L'échec de la traduction de Moussa Kamara, "l'impossible traduction» (Schmitz dans Schmitz \& Bousbina 1998 : 30), correspondait précisément à la fin de la « saison orientaliste » dont nous venons de parler, et dont elle signait elle aussi la clôture. « La mort de Delafosse [...] en 1926, remet tout en cause dans la mesure où, avec Gaden, ils formaient une véritable équipe $[. .] ».($ ibid.).

Si les Ta'rîkh-s ont été le point de fixation des travaux orientalistes du tournant des XIX et $\mathrm{Xx}^{\mathrm{e}}$ siècles pour la boucle du Niger, l'œuvre de Moussa Kamara joue un rôle un peu analogue pour la vallée du fleuve Sénégal ${ }^{56}$. Les publications faites à l'IFAN, dans les années 1970, et les travaux sur l'histoire de la vallée du fleuve Sénégal, notamment 
ceux de David Robinson, et, plus spécifiquement son article dans les Cahiers d'Études africaines, en 1988, avaient ramené l'attention sur cette somme historique ${ }^{57}$.

Somme s'il en est ${ }^{58}$ : la version manuscrite en deux tomes, déposée au département d'islamologie de l'IfAN-Université Cheikh Anta Diop de Dakar, compte 867 folios. Jean Schmitz, anthropologue de la vallée du Sénégal, s'est fait chef de chantier et a mobilisé des arabisants et des foulanisants, des historiens et des anthropologues, avec une équipe à Paris, et l'autre à Dakar ${ }^{59}$, pour la traduction de la partie jugée prioritaire concernant le Futa Toro, soit 688 folios.

La traduction proprement dite, en français, de ces 688 folios a été achevée par les deux équipes qui se sont mises au travail à partir de janvier 1990 (Paris) et décembre 1990 (Dakar). La programmation prévoit cinq volumes à paraître dont le premier, traduit par Said Bousbina, et accompagné par un riche appareil anthropologique et historique établi par Jean Schmitz, a été publié en 1998 (ibid.). Il couvre 153 folios de texte ${ }^{60}$, soit un petit quart du total « utile» (les 688 folios sur le Futa Toro), et le sixième du Zuhûr entier. Le texte arabe accompagne ce volume 1 sous la forme de quatre microfiches jointes à la quatrième de couverture - solution de compromis entre la reproduction imprimée des textes arabes et leur absence.

89 Compte tenu de l'architecture complexe de la numérotation des tomes, des cahiers et des chapitres de l'original et de celle des volumes à publier, ce bilan chiffré permet de rendre compte du travail effectué, et de celui qu'il reste à faire ${ }^{61}$.

Pour ces raisons, on pourrait craindre que le complexe de l'« impossible traduction » frappe à nouveau pour l'achèvement de la publication. Quoi qu'il advienne, il reste un volume important qui a fait avancer l'exploitation d'une source arabo-africaine majeure, en reprenant à sa manière l'héritage de Gaden et Delafosse. On notera que la démarche n'est plus exclusivement "orientaliste " (l'édition savante d'une source), mais immédiatement intégrée dans un agenda d'anthropologie historique, avec un intérêt particulier pour les généalogies, les mariages, les lignées utérines, les relations entre affins, qui dépasse donc les enjeux d'une annotation de type orientaliste. Ainsi se trouve rétablie une forme de lien entre islamologie et ethnologie qui, après Delafosse, s'était largement distendue, mais il y faut maintenant toute une équipe.

91 La veine orientaliste se poursuit encore dans d'autres travaux, notamment ceux de Constant Hamès (1987, 1993, 1997a, 1997b, 2007, 2008) qui, parallèlement à Louis Brenner (1984, 1985), a ouvert un chantier neuf, dans un domaine négligé, sinon déprécié, celui de la magie en islam : "Les deux ou trois générations de ceux qu'on a appelés orientalistes sont, dans l'ensemble, restés bien silencieuses sur le sujet» (Hamès 2008 : 95) et, ajoute-t-il, « Du côté de l'Afrique, la recherche dans ces domaines reste inhibée ${ }^{62}$ et a certainement devant elle des perspectives considérables de découverte (données manuscrites et de terrain) [...]» (ibid. : 99). G. Anawati (1972) avait ouvert cette voie dans une édition orientaliste du Caire à partir d'un talisman de Mopti (Mali).

92 Les autres successeurs doivent être recherchés dans le monde académique anglophone. En 1999, John Hunwick publie une nouvelle traduction du Ta'rîkh al-Sûdân, véritable édition du centenaire (1899-1999). L'édition Houdas-Benoist avait terriblement vieilli. Les notes, en particulier, correspondaient à un niveau de connaissances largement dépassé. C'est une édition critique, accompagnée de quelques textes connexes, dont les notes, attentives à tous les faits d'histoire subsaharienne et de linguistique arabe, soninké et songhay, représentent l'un des derniers états des connaissances. Cette 
traduction s'intègre dans une présentation de l'histoire de l'empire songhay, fidèle au texte, mais dont nous allons voir qu'elle va être rapidement débordée par un autre ouvrage majeur, celui de P.F. de Moraes Farias. Le texte arabe est absent, ce que regrette l'auteur, mais les éditeurs ne sont plus en mesure d'en assurer les coûts. J. Hunwick est, par ailleurs, avec R. S. O'Fahey, le maître d'œuvre d'un vaste inventaire des sources arabes subsahariennes, Arabic Literature of Africa (ALA), qui se présente dans la lignée d'instruments d'identification des auteurs et œuvres arabes, bien connus des orientalistes : Carl Brockelmann, Geschichte der arabischen Literarur (1937-1949) et Fuat Sezgin, Geschichte des arabischen Schrifttums (1967 sq.), remise en chantier du précédent ${ }^{63}$.

93 Signe de l'absence totale des "périphéries» dans ce bagage orientaliste, le Brockelmann ne comptait pas plus de cinq pages sur les auteurs et les œuvres hors du Maghreb et de l'Égypte. Quant au Sezgin, il en était encore, en 1994, après dix volumes, aux cinq premiers siècles de l'islam. Si l'on y ajoute les dictionnaires biographiques : le Al-A'lam de Khayr al-Dîn al-Ziriklî (1927, rééd. et mises à jour) et le Mu'jam al-Mu'allifîn, dictionnaire d'auteurs, de 'Umar Ridâ Kahhâla (1957-1961), le bilan est identique. « La consultation de ces quatre références majeures [...] donne l'impression que l'Afrique subsaharienne n'a pas contribué à l'histoire intellectuelle du monde musulman » (Kane 2003: 6). Il y avait donc là un extraordinaire vide, aussi bien dans les instruments arabes que dans les corpus occidentaux.

Arabic Literature of Africa publiée par Brill, comme Brockelmann et Sezgin, compte six volumes couvrant la haute vallée du Nil, l'Afrique orientale, la Corne de l'Afrique, l'Afrique occidentale et le Sahara de l'Ouest. Le volume qui nous intéresse ici plus particulièrement est le volume 4, The Writings of Western Sudanic Africa (Hunwick 2003) ${ }^{64}$. Cette entreprise éditoriale se double d'une opération de sauvegarde des manuscrits de Tombouctou, engagée en 2004, et préparée par des actions antérieures. Ce projet américano-norvégo-malien, soutenu par l'ISITA, Institute for the Study of Islamic thought in Africa (Northwestern University), financé par la fondation Ford, a pour but de numériser « 50000 manuscrits » arabo-africains de la zone, en accompagnant cette opération d'actions culturelles et éditoriales ${ }^{65}$.

On signalera enfin une autre entreprise, en langue arabe, qui a également des retombées sur Tombouctou. Créée au début des années 1990 par le cheikh Ahmed Zaki Yamani, ancien ministre séoudien du Pétrole, la Fondation Al-Furqân li-Ihya al-Turath al-Islamî (Al-Furqan Islamic Heritage) basée à Londres, s'assigne pour tâche de revivifier le patrimoine culturel islamique. Après la publication, chez Brill, d'une encyclopédie en quatre volumes de collections de manuscrits dans la majorité des pays musulmans (Roper 1992-1994), qui comprend de nombreuses entrées relatives aux pays d'Afrique subsaharienne, une trentaine de catalogues en arabe portant sur des collections non cataloguées ont été publiés. Près de la moitié concernent plusieurs pays africains (Kane 1997, pour le Sénégal).

D'autre part, la collaboration entre al-Furqan et le CEDRAB (devenu IHERIAB, Institut des hautes études et de recherches islamiques Ahmed Baba) de Tombouctou a permis la publication en arabe de cinq volumes de catalogues (Kane $2003: 10-11$ ), soit près de 5000 manuscrits, compilés par des bibliothécaires du CEDRAB et des spécialistes étrangers (Ould Eli \& Johansen $1995^{66}$; Haidara \& Ayman Fuad Sayyid 200067).

Ainsi, Tombouctou et la boucle du Niger reprennent-elles l'avantage dans ces nouveaux dispositifs «mondialisés ». Là, se trouve l'héritage le plus direct de la voie ouverte par 
Houdas, Benoist et Delafosse. Avec eux, le cycle Houdas-Delafosse s'efface au profit de nouveaux travaux orientalistes qui en reprennent le fil. Un bel ouvrage, issu d'une conférence sur le Tombouctou Manuscript Project de l'Université du Cap, tenue en août 2005, vient couronner pour un temps l'édifice de cette nouvelle recherche tombouctienne. Il s'agit du volume publié par Shamil Jeppie et Souleymane Bachir Diagne, The Meanings of Timbuktu (2008), qui réunit, avec 24 communications de chercheurs africains, américains et européens, et de riches illustrations, les principaux acteurs du renouveau des études sur les textes manuscrits subsahariens en général, et sur l'histoire et la production intellectuelle de la boucle du Niger en particulier. On y trouve un bilan global de l'état de la question.

Mais l'ouvrage qui a profondément bouleversé les études sur la boucle du Niger, et qui, intellectuellement, ferme la marche pour le moment, est celui de Paulo Fernando de Moraes Farias (2003), véritable événement historiographique qui, à partir d'une collecte d'inscriptions arabes pour la plupart inédites, recueillies dans l'est de la boucle du Niger, en remontant jusqu'à l'Adrar des Ifoghas, révolutionne l'histoire de l'introduction de l'islam dans la zone, et reconstruit, sur le plan de l'analyse historique, la vulgate des $T a^{\prime}$ rîkh. Les premières inscriptions datent $\mathrm{du} \mathrm{XI} \mathrm{I}^{\mathrm{e}}$ siècle, et constituent donc les sources écrites les plus anciennes sur la région. Nous nous sommes déjà longuement exprimé sur cet ouvrage (Triaud 2004, 2005). Nous n'y reviendrons pas, sinon pour redire que ces sources épigraphiques, ainsi que la réintégration, par Moraes Farias, des récits culturels et des traditions orales de la région dans l'analyse historique, ont donné un " coup de vieux » à nombre de travaux historiques sur cette partie du Sahel. C'est comme si, après un long temps de fascination pour les seuls Ta'rîkh, l'histoire, toute l'histoire, reprenait ses droits et échappait finalement à l'emprise de la première strate orientaliste, celle de Houdas et Delafosse, qui, en privilégiant les Ta'rîkh, avaient nolens volens validé une histoire apologétique et idéologique.

La démarche "positiviste » de Delafosse (Triaud 1998b) avait été piégée par ces belles séries dynastiques songhay successives et datées, dont la partie la plus haute, au moins, répondait à une construction visant à donner aux occupants marocains (Armas) une image flatteuse de la monarchie songhay dans la longue durée. Les Ta'rîkh gardent toute leur importance, mais sous un éclairage profondément renouvelé. Ce dépassement des Ta'rîkh et de l'œuvre de traduction du tandem Houdas-Delafosse par les éditions de Hunwick et de Moraes Farias marque bien la fin d'un cycle.

101 Si l'on considère le processus de façon rétrospective, les relations avec l'establishment orientaliste du nord du Sahara ont été constamment, pour la période qui nous intéresse, marquées d'ambiguïtés. Les experts orientalistes restent les instances d'appel, mais l'initiative et la décision reviennent désormais aux «Soudanais ». C'est l'émergence d'une science "soudanaise » distincte de la «nord-africaine » qui est en jeu. En d'autres termes, l'orientalisme subsaharien a utilisé les flux venus du Maghreb pour émerger, puis s'en est éloigné pour échapper à son emprise et construire ses propres représentations ${ }^{68}$.

102 Les administrateurs-savants, à la recherche de cette différenciation, vont désormais quêter leur consécration scientifique, d'une part dans des supports locaux, comme le Bulletin du Comité d'Études historiques et scientifiques de l'AoF, véritable organe de l'autonomie scientifique, qui commence sa publication en 1918, et, d'autre part, dans les revues d'anthropologie et d'ethnologie de la métropole, quoique, en matière d'islam, 
il ne semble pas que cela ait représenté un débouché majeur. Si l'on consulte la bibliographie de Delafosse, on constate que les écrits sur l'islam et les sociétés musulmanes vont à la Revue du Monde musulman et au Bulletin du Comité de l'Afrique française, tandis que ceux sur les langues et les religions africaines sont destinés à différentes revues d'ethnographie (Amselle \& Sibeud 1998: 307-319). Ainsi, se reproduit, en même temps, la partition déjà signalée entre une ethnologie qui s'occupe des religions locales, et une étude de l'islam destinée à d'autres publics. Delafosse est à la jointure des deux pour un temps. Quand il disparait, les deux démarches vont poursuivre leur chemin séparément.

En dépit de cette autonomisation d'un orientalisme "soudanais ", il reste un ancrage commun puissant, là où se retrouvent, même si ce n'est pas toujours en bonne intelligence, les "Soudanais» et les «Nord-Africains ». Il s'agit de l'alma mater, véritable point de ralliement de ces études, que constitue l'École des Langues orientales, à Paris. Là, s'enseignent les arabes littéral et dialectal, et les grandes langues de l'islam subsaharien: le manding, le peul et le hausa, ainsi que le wolof. Tous les chemins de l'étude de l'islam subsaharien, et, plus particulièrement, de l'orientalisme, passent donc par elle.

Une dernière phase doit enfin être considérée, celle de l'appropriation de cet héritage par les chercheurs africains. C'est une nouvelle quête d'autonomie qui se dessine alors. Amadou Hampâté Bâ, l'auteur de L'Empire peul du Macina (1955) en est la figure de proue. Il y impose une méthode propre d'histoire africaine entièrement fondée sur des sources orales collectées et mises en récit.

On doit à un ouvrage de Kusum Aggarwal (1999), universitaire indienne, et au compte rendu stimulant qu'en a fait Bernard Mouralis (2000a), des pistes de réflexion qui nous conduisent, avec Hampâté Bâ, au terme de ce parcours. K. Aggarwal (1999 : 217) voit dans L'Empire peul, un « véritable manifeste historique [qui] montre pour la première fois comment et de quelle manière les Africains se pensent eux-mêmes en relation avec les moments fondateurs de leur passé historique" (1999: 203). "L'itinéraire de Hampâté Bâ peut être considéré - ajoute-t-elle - comme un processus de "reprise de la signature" ".

Kusum Aggarwal (ibid.: 242) évoque encore une « reconquête du champ scientifique à part entière " par les auteurs africains, dont Hampâté Bâ serait un bon exemple : non pas par un contre-discours, mais par un « mouvement vers l'autonomisation de la voix auctoriale ( (ibid.: 240). «[Aggarwal] montre aussi comment Hampâté Bâ tente de construire une histoire africaine en s'éloignant de la vision souvent anhistorique que l'africanisme donnait de l'Afrique; mais, ce faisant, s'il s'éloigne de Griaule, il se rapproche de Delafosse en cherchant comme lui "à inventer une Afrique africaine, épurée du regard extérieur des voyageurs, des explorateurs, des colonisateurs" (ibid.: 203)» (Mouralis 2000a : 379).

107 Décidément, Delafosse reste incontournable, un Delafosse sans doute idéalisé dans ce cas. Du moins, Houdas, Delafosse et Gaden ont-ils cherché, avec leurs moyens de l'époque, à dépasser ce regard extérieur, en puisant dans les œuvres écrites « indigènes » disponibles (Ta'rîkh-s et Siré Abbas Soh), ou en les suscitant (Moussa Kamara), procédant ainsi à une valorisation des sources proprement africaines, à un décentrement du savoir islamologique accumulé au Maghreb et à une recomposition autonome sous la forme d'un orientalisme proprement soudano-sahélien. 


\section{BIBLIOGRAPHIE}

ABITBOL, M. (dir.), 1982 Tombouctou au milieu du XVIII siècle d'après la chronique de Mawlay al Qasim $b$. Mawlay Sulayman (prés. et trad. de l'arabe), Paris, Maisonneuve.

AGGARWAL, K., 1999 Amadou Hampâté Bâ et l'africanisme. De la recherche anthropologique à l'exercice de la fonction auctoriale, Paris, L'Harmattan.

AL-OMARI, Ibn Fadl Allah, 1927 Masâlik El Absâr fi Mamâlik El Amsâr. I. L'Afrique, moins l'Égypte (trad. et annoté par Gaudefroy-Demombynes), Paris, Paul Geuthner.

AL-ZIRIKLî, K. al-D., 1927 Al-A'lam : qamus tarajim li-ash'har al-rijal wa-al-nisa' min al-Arab wa-almustashriqin, Éditions variées (rééd. 1957, 1969 1979).

AMSELLE, J.-L., 1990 Logiques métisses. Anthropologie de l'identité en Afrique et ailleurs, Paris, Payot (nouvelle éd., 1999).

-, 1996 Vers un multiculturalisme français : l'empire de la coutume, Paris, Flammarion (nouvelle éd.

«Champs », 2001).

AMSELLE, J.-L. \& SIBEUD, E. (dir.), 1998 Maurice Delafosse. Entre orientalisme et ethnographie : l'itinéraire d'un africaniste (1870-1926), Paris, Maisonneuve et Larose.

ANAWATI, G., 1972 « Trois talismans en arabe provenant du Mali (marché de Mopti) », Annales islamologiques, $11: 287-339$.

ARNAUD, R., 1912 « L'islam et la politique musulmane française en Afrique occidentale française », Bulletin du Comité de l'Afrique française, Renseignements coloniaux, 1 : 3-20 ; 3 : 115-127, 142-154, Paris, Publication du Comité de l'Afrique française.

BA, A.-H. \& DAGET, J., 1955 L'Empire peul du Macina, t. 1, 1818-1853, Bamako, IFAN, Études soudanaises (rééd. Paris-La Haye, Mouton, 1962 ; Abidjan, Nouvelles Éditions africaines-Éditions de l'EHESS, 1984).

BARRows, L. C., 1977 «Faidherbe's Islamic Policy in Senegal », French Colonial Studies, 1 : 34-53.

BASSET, R., 1883 « Catalogue de deux bibliothèques de Fès (al-Qarawiyyîn et al-Rasif) », Bulletin de Correspondance africaine, fasc. VI : 366-393.

-, 1909-1913 Mission au Sénégal, I : Étude sur le dialecte zénaga, II : Notes sur le hassania, III :

Recherches historiques sur les Maures, Paris, E. Leroux (« Bulletin de Correspondance africaine », t. XXXIX).

BOUSBINA, S., 1993 « L'histoire de l'almaami Abdul (1727-1806), par Shaykh Muusa Kamara », (trad. de S. Bousbina ; prés. de J. Schmitz), Islam et Sociétés au sud du Sahara, 7 : 59-93.

BousBinA, S., SCHMiTZ, J. \& PONDOPOULO, A., 1993 « À la découverte de Shaykh Musa Kamara », Islam et Sociétés au sud du Sahara, $7: 57-109$.

BRENNER, L., 1984 West African Sufi : the Religious Heritage and Spiritual Search of Cerno Bokar Saalif Taal, London, Hurst ; Berkeley, University of California Press.

-, 1985 Réflexions sur le savoir islamique en Afrique noire, Bordeaux, Centre d'Étude d'Afrique noire.

-, 2000 « Amadou Hampâté Bâ, Tijâni francophone », in J.-L. TRIAUD \& D. ROBINSON (dir.), La

Tijâniyya. Une confrérie musulmane à la conquête de l'Afrique, Paris, Karthala : 289-326. 
BROCKELMANN, C., 1937-1949 Geschichte der arabischen Literarur, Leiden, Brill, 2 vol.

CONKLIN, A., 1997 A Mission to Civilize : the Republican Idea of Empire in France and West Africa, 1895-1930, Stanford, Stanford University Press.

CUOQ, J., 1975 Recueil des sources arabes concernant l'Afrique occidentale du VIII ${ }^{e}$ au XVI ${ }^{e}$ siècle (Bilâd alSûdân), Paris, Éditions du CNRS.

DELAFOSSE, L., 1976 Maurice Delafosse. Un Berrichon conquis par l'Afrique, Paris, Société française d'Histoire d'Outre-Mer.

DELAFOSSE, M., 1900 Essai de manuel de la langue agni (dialecte baoulé), Paris, Librairie africaine et orientale Joseph André.

-, 1901a Essai pratique de la langue mandé ou mandingue, Paris, E. Leroux.

-, 1901b Manuel de langue haoussa ou chrestomathie haoussa, précédé d'un abrégé de grammaire et suivi d'un vocabulaire, Paris, Maisonneuve.

-, 1910a « L'état actuel de l'islam dans l'Afrique occidentale française », Revue du Monde musulman, $11: 32-53$

—, 1910b « Le clergé musulman de l'Afrique occidentale », Revue du Monde musulman, 11 : 177-206.

-,1910c « Coutumes et cérémonies matrimoniales chez les musulmans de l'Afrique occidentale », Revue du Monde musulman, 11 : 405-421.

,$- 1910 d$ « Le nom des Noirs musulmans du Soudan occidental », Revue du Monde musulman, 12 : 257-261.

-, 1911 « Les confréries musulmanes et le maraboutisme dans les pays du Sénégal et du Niger », Bulletin du Comité de l'Afrique française, Renseignements coloniaux, 4 : 81-90.

-, 1912 La politique musulmane française en Afrique occidentale française, Paris, Publication du Comité de l'Afrique française.

-, 1913 « Traditions historiques et légendaires du Soudan ancien, traduites d'un manuscrit arabe inédit », Bulletin du Comité de l'Afrique française, Renseignements coloniaux : 293-306, 325-329, 355-368.

-, 1929 La langue mandingue et ses dialectes (Malinké, Bambara, Dioula), t. I, Introduction, grammaire, lexique français-mandingue, Paris, Paul Geuthner.

-, 1955 La langue mandingue et ses dialectes (Malinké, Bambara, Dioula), t. II, Dictionnaire mandinguefrançais, Paris, Paul Geuthner.

DELAFOSSE, M. \& GADEN, H., 1913 «Chroniques du Foûta sénégalais », Revue du Monde musulman, XXIV : 1-114, XXV : 165-235.

DEPONT, O. \& COPPOLANI, X., 1897 Les confréries religieuses musulmanes, Alger, Adolphe Jourdan. DIALLO, T., M'BACKÉ, M. B., TRIFKOVIC, M. \& BARRY, B., 1966 Catalogue des manuscrits de l'IFAN, Dakar, IFAN.

DIDIER, H., 1997 « Louis Massignon et Charles de Foucauld », in J. KERYELL (dir.), Louis Massignon et ses contemporains, Paris, Karthala : 93-109.

DUMONT, F., 1975 La pensée religieuse d'Amadou Bamba, Dakar, Nouvelles Éditions africaines.

EL-BEKRI, A.-O., 1911-1913 Description de l'Afrique septentrionale (trad. de W. Mac Guckin de Slane), Alger, Geuthner., 1968 «Al-Bakrî (Cordoue 1068), routier de l'Afrique blanche et noire du nord-ouest » 
(trad. nouvelle de seize chapitres avec notes et commentaire par Vincent Monteil), Bulletin de l'IFAN, B, 30 (1) : 39-116.

EL HAMEL, Ch., 1994 « Biographie de Muhammad b. Abû Bakr as-Siddiq Al-Burtilî al-Walatî », Islam et Sociétés au sud du Sahara, $8: 85-93$.

-, 2002 La vie intellectuelle islamique dans le Sahel ouest-africain (XVI ${ }^{e}$-XIX ${ }^{e}$ siècles), (trad. de Fath ashshakur d'al-Bartili al-Walati), Paris, L'Harmattan.

ES-SA'DI, A., 1898-1900 Tarikh Es-Soudan, par Abderrahman ben Abdallah ben' Imran ben 'Amir Es-Sa'di, Texte arabe éd. et trad. par O. Houdas avec la coll. de Edm. Benoist.

FAIDHERBE, L., 1856 « Populations noires des bassins du Sénégal et Haut Niger », Revue coloniale, $2^{\mathrm{e}}$ série, 16 , octobre : 328-341.

-, 1877 Le zénaga des tribus sénégalaises : contribution à l'étude de la langue berbère, Paris, E. Leroux. FRÉBOURG, C., 1990 Le Corse en Mauritanie : Xavier Coppolani (1866-1905). L'islam au service de la France, Mémoire de maîtrise, Paris, Université Paris VII.

FRÉMEAUX, J., 1998 « Les Bureaux arabes et Delafosse. Contribution à une étude de l'historiographie coloniale », in J.-L. AMSELLE \& E. SIBEUD (dir.), op. cit. : 193-209.

FROELICH, J.-C., 1962 Musulmans d'Afrique noire, Paris, Éditions de l'Orante.

GADEN, H., 1912 « Légendes et coutumes sénégalaises : les Cahiers de Yoro Dyao », Revue d'ethnographie et de sociologie, 3-4 : 119-137, 191-202.

-, 1913-1914 Le Poular, dialecte peul du Fouta sénégalais, vol. I, Étude morphologique ; vol. II, Lexique poular-français, Paris, E. Leroux., 1931 Proverbes et maximes peuls, et toucouleurs, traduits, expliqués, annotés, Paris, Institut d'Ethnologie (« Travaux et Mémoires », 16)., 1935 La vie d'El Hadj Omar. Qacida en poular de Mohammadou Aliou Tyam, (transcription, traduction, notes et glossaire), Paris, Institut d'Ethnologie.

-, 1969-1972 Dictionnaire peul-français (transcription, traduction, notes et glossaire), Dakar, Catalogues et documents de l'IFAN, 2 fasc.

GHALI, N., MAHIBOU, S.-M. \& BRENNER, L., 1985 Inventaire de la Bibliothèque 'umarienne de Ségou, Paris, Éditions du CNRS.

GLASMAN, J., 2002 La classification ethnique du Sénégal, Mémoire de maîtrise, Aix-en-Provence, Université de Provence. <http://sites.univ-provence.fr/wclio-af/d_fichiers11/sommaire.html>.

-, 2004 « Le Sénégal imaginé. Évolution d'une classification ethnique de 1816 aux années 1920 ", Afrique \& histoire, 2 (1) : 111-139. <http://www.cairn.info/article_p.php ?

ID_ARTICLE=AFHI_002_0111>.

HAIDARA, A. M. (comp.) \& AYMAN FUAD SAYYID (eds.), 2000 Catalogue of Manuscripts in Mamma Haidara Library, London, Al-Furqân, Islamic Heritage Foundation, 3 tomes.

HAMES, C., 1987 « Taktub ou la magie de l'écriture islamique. Textes soninké à usage magique », Arabica, XXXIV : 305-335., 1993 « Entre recette magique d'al-Buni et prière islamique d'alGhazali : textes talismaniques d'Afrique occidentale », in A. DE SURGY (dir.), Fétiches II. Puissance des objets, charme des mots, Paris, Systèmes de Pensée en Afrique noire, Cahier 12 : 187-233.

-, 1997a L'art talismanique en islam d'Afrique occidentale. Personnes, supports, procédés, transmission. Analyse anthropologique et islamologique d'un corpus de talismans à écriture, Thèse de doctorat, Paris, École pratique des hautes études. 
,$- 1997 \mathrm{~b}$ « Le Coran talismanique. De l'Arabie des origines à l'Afrique occidentale contemporaine. Délimitation et inventaire des textes et des procédés linguistiques utilisés », in A. DE SURGY (dir.), Religion et pratiques de puissance, Paris, L'Harmattan : 139-160.

-, 2007 Coran et talismans. Textes et pratiques magiques en milieu musulman, Paris, Karthala.

-, 2008 « Problématiques de la magie-sorcellerie en islam et perspectives africaines », Cahiers d'Études africaines, 48 (1-2), 189-190: 81-99.

HAMET, I., 1906 Les musulmans français du nord de l'Afrique, Paris, Armand Colin.

-, 1911 Chroniques de la Mauritanie sénégalaise. Nacer Eddine, Paris, E. Leroux.

-, 1923 Histoire du Maghreb, Cours professé à l'Institut des Hautes études marocaines, Paris, E. Leroux.

HEYMOWSKI, A. \& OULD HAMIDOUN, M., 1965-1966 Catalogue provisoire des manuscrits mauritaniens en langue arabe préservés en Mauritanie, Nouakchott-Stockholm.

HILLIARD, C., 1987 « Al-Majmû' al-Nafîs : Perspectives on the Origins of the Muslim Torodbe of Senegal from the Writings of Shaikh Musa Kamara », Islam et Sociétés au sud du Sahara, 11 : 175-186.

HISKETT, M., 1975 A History of Hausa Islamic Verses, London, SOAS.

HOUDAS, O., 1886 Ethnographie de l'Algérie, Paris, Maisonneuve et Leclerc.

-, 1897 Précis de grammaire arabe, Paris, Librairie africaine et orientale, Joseph André.

-, 1898-1900 Tarikh es-Soudan, par Abderrahman ben Abdallah ben' Imran ben 'Amir es-Sa'di, Paris, E. Leroux (éd. par O. Houdas avec la collaboration d'E. Benoist).

-, 1899-1901 Tedzkiret en-Nisiân fi Akhbâr Molouk al-Soudan, Paris, E. Leroux (éd. par O. Houdas avec la collaboration d'E. Benoist)., 1904 L'Islamisme, Paris, Dujarric.

HOUDAS, O. \& DELAFOSSE, M. (dir.), 1913 Tarikh el-Fettach fi Akhbâr El-Bouldân Oua-L-Djouyouch OuaAkâbir En-Nâs, ou Chronique du chercheur pour servir à l'histoire des villes, des armées et des principaux personnages du Tekrour, par Mahmoûd Kâti ben El-Hâdj El-Motaouakkel Kâti et l'un de ses petitsfils, Paris, Adrien Maisonneuve.

HOUDAS, O. \& MARÇAIS, W. (dir.), 1903-1914 El-Bokhari (Muhammad), Les traditions islamiques, Paris, E. Leroux, 4 vol. (rééd. Adrien-Maisonneuve, 1977).

HUNWICK, J., 1999 Timbuktu \& the Songhay Empire. Al-Sa'd'dî’s Ta'rîkh al-Sûdân down to 1613 \& other Contemporary Documents, Leiden, Brill.

-, 2003 Arabic Literature of Africa, vol. 4, The Writings of Western Sudanic Africa, Leiden, Brill. HUNWICK, J. \& HARRAK, F., 2000 Mi'raj al-Su'ud: Ahmad Baba's Replies on Slavery, Rabat, Université Mohammed V, Institut d'Études africaines (« Textes et documents »).

IBN KHALDOUN, 1852-1856 Histoire des Berbères et des dynasties musulmanes de l'Afrique Septentrionale (trad. par William Mac Guckin de Slane), Alger, Imp. du Gouvernement, 4 vol.

JEPPIE, S. \& DIAGNE, S. B., 2008 The Meanings of Timbuktu, Cape Town, HSRC Press.

KAHHÂLA, 'U. R., 1957-1961 Mu'jam al-mu'allifîn : tarâjim musannifin al-kutub al-'arabiyya, 15 vol., Damascus.

KAMAL, Y., 1926-1951 Monumenta Cartographica Africae et Aegypti, Le Caire-Leyde, publié par l'auteur, 16 vol. 
KAMARA, S.-M., 1970 « La vie d'El Hadj Omar » (éd. et trad. par Amar Samb), Bulletin de l'IFAN, B, 32 (1) : 44-135.

-, 1973 «L'islam et le christianisme » (éd. et trad. par A. Samb), Bulletin de l'IFAN, B, 35 (2) : 269-322.

-, 1975 « Histoire du Boundou » (éd. et trad. par M. Ndiaye), Bulletin de l'IFAN, B, 37 (4) : 784-816.

-, 1976 « La condamnation de la guerre sainte » (éd. et trad. par A. Samb), Bulletin de l'IFAN, B, 38 (1) : 158-199.

-, 1978 « Histoire de Ségou » (éd. et trad. par M. Ndiaye), Bulletin de l'IFAN, B, 40 (3) : 458-488.

-, 1993 « L'histoire de l'almaami Abdul (1727-1806), par Shaykh Muusa Kamara » (trad. de Saïd Bousbina, prés. de Jean Schmitz), Islam et Sociétés au sud du Sahara, 7 : 60-93.

KANE, O., 1997 Handlist of Arabic Manuscripts, Sénégal, London, Al-Furqan, Islamic Heritage Foundation.

-, 2003 Intellectuels non europhones, Dakar, Codesria.

<http://www.codesria.org/Links/Publications/monographs/ousmane_kane.pdf>.

KA'TI, M. \& l'un de ses petits-fils, 1913 Tarikh el-Fettach fi Akhbâr El-Bouldân Oua-L-Djouyouch OuaAkâbir En-Nâs, ou Chronique du chercheur pour servir à l'histoire des villes, des armées et des principaux personnages du Tekrour par Mahmoûd Kâti ben El-Hâdj El-Motaouakkel Kâti et l'un de ses petitsfils, Paris, Adrien Maisonneuve.

KUCZYNSKI, L., 2002 Les marabouts africains à Paris, Paris, Éditions du CNRS.

KYBURZ, O., 1994 « La littérature peule dans la collection "Classiques africains" ", Cahiers d'Études africaines, XXXIV (1-3), 133-135 : 483-488.

LACRoIX, P.-F., 1965 Poésie peule de l'Adamawa, Paris, Julliard, 2 vol.

LANGE, D., 1977 Chronologie et histoire d'un royaume africain, Wiesbaden, Franz Steiner.

LE CHATELIER, A., 1887 Les confréries musulmanes du Hedjaz, Paris, E. Leroux (« Bibliothèque orientale elzévirienne »).

-, 1899 L'islam dans l'Afrique occidentale, Paris, G. Steinheil.

LE CHATELIER, J., 1987 Alfred Le Chatelier, 1855-1929 : sa carrière africaine, Paris, Service historique de l'Armée de Terre.

LEVTZION, N. \& HOPKINS, J. F. K., 1981 Corpus of Early Arabic Sources for West African History, Cambridge, Cambridge University Press.

MAHIBOU, S. M. \& TRIAUD, J.-L., 1983 Voilà ce qui est arrivé. Bayân mâ waqa’a d’al-Hâjj ‘Umar al-Fûtî. Plaidoyer pour une guerre sainte, en Afrique de l'Ouest au XIX ${ }^{e}$ siècle, Paris, Éditions du CNRS.

MARCHAND (Interprète), 1897 «La religion musulmane au Soudan français », Bulletin du Comité de l'Afrique française, Renseignements coloniaux, octobre : 91-111.

MARTY, P., 1913 Les Mourides d'Amadou Bamba, Paris, E. Leroux.

-, 1915-1916 L’Islam en Mauritanie et au Sénégal, Paris, E. Leroux.

-, 1916 Études sur l'islam maure: Cheikh Sidia, les Fadelia, les Ida Ou Ali, Paris, E. Leroux (« Collection de la Revue du Monde musulman »). 
-, 1917 Études sur l'Islam au Sénégal, Paris, E. Leroux («Collection de la Revue du Monde musulman »), 2 vol., 1920 " M’Hammed Ould Ahmed Youra, "Le Livre des Lettrés renseignés sur l'histoire des puits" ", (trad.), Bulletin du Comité d'Études historiques et scientifiques de l'AOF, III (3) : 311-345.

-, 1920-1921 Études sur l'Islam et les tribus du Soudan, Paris, E. Leroux (« Collection de la Revue du Monde musulman »), 4 vol.

-, 1921 L'Islam en Guinée : Fouta Diallon, Paris, E. Leroux («Collection de la Revue du Monde musulman »).

-, 1922 Études sur l'Islam en Côte-d'Ivoire, Paris, E. Leroux («Collection de la Revue du Monde musulman »)., 1926 Études sur l'Islam au Dahomey, Paris, E. Leroux (« Collection de la Revue du Monde musulman »).

-, 1930-1931 « L'Islam et les tribus dans la Colonie du Niger », Revue des Études islamiques, 1930 :

$333-349 ; 1931: 139-240$.

MASSIGNON, L., 1909 « Une bibliothèque saharienne : la bibliothèque du Cheikh Sidia », Revue du Monde musulman, VIII : 409-418.

MBACKÉ, K. \& KA, T., 1994 « Nouveau catalogue des manuscrits de l'IFAN », Islam et Sociétés au sud du Sahara, $8: 165-199$.

MBAYE (El-Hadji R.) \& MBAYE, B., 1975 « Supplément au catalogue des manuscrits de l'IFAN », Bulletin de l'IFAN, B, 37 : 878-895.

MONTEIL, V., 1964 L’Islam noir, Paris, Éditions du Seuil (rééd. 1971, 1980).

-, 1965-1967 « Les manuscrits historiques arabo-africains. Bilan provisoire », Bulletin de l'IFAN, B, 27 : 531-542 ; B, 28 : 668-675 ; B, 29 : 599-603.

-, 1967 Esquisses sénégalaises, Dakar, IFAN.

-, 1968 «Al-Bakrî (Cordoue 1068), Routier de l'Afrique blanche et noire du Nord-Ouest » (trad. nouvelle de seize chapitres avec notes et commentaire), Bulletin de l'IFAN, B, 30 :39-116.

DE MORAES FARIAS, P. F., 2003 Arabic Medieval Inscriptions from the Republic of Mali : Epigraphy, Chronicles and Songhay-Tuareg History, Oxford, Oxford University Press.

MOURALIS, B., 2000a Compte rendu du livre de K. AGGARWAL, Amadou Hampâté Bâ et l'africanisme. De la recherche anthropologique à l'exercice de la fonction auctoriale, in Cahiers d'Études africaines, XL (2), $158: 377-379$.

,$- 2000 b$ « Orientalisme et africanisme : réflexion sur deux objets », in P. S. DIOP \& H.-J. LÜSEBRINK (dir.), Littératures et sociétés africaines. Regards comparatistes et perspectives interculturelles, mélanges offerts à János Riesz, Tübingen, Gunter Narr : 17-28.

NDIAYE, M., 1976 « Histoire du Boundou » (éd. et trad. par M. Ndiaye), Bulletin de l'IFAN, B, 37 (4) : 784-816.

-, 1978 « Histoire de Ségou » (éd. et trad. par M. Ndiaye), Bulletin de l'IFAN, B, 40 (3) : 458-488.

OULD CHEIKH, A. W., 1987 « Rohkatalog der arabischen Handschriften in Mauretanien », Islam et Sociétés au Sud du Sahara, $1: 109-113$.

OULD ELI, Sidi Amar (comp.) \& JOHANSEN, J. (eds.), 1995 Handlist of Manuscripts in the Centre de Documentation et de Recherches Ahmad Baba, Timbuktu, Mali, vol. I-V, London, al-Furqan Islamic Heritage Foundation. 
PASQUIER, R., 1974 «L'influence de l'expérience algérienne sur la politique de la France au Sénégal (1842-1869) », in Mélanges Hubert Deschamps, Paris, Publications de la Sorbonne : 263-284.

PONDOPOUlO, A., 1993 « Une traduction “mal partie" (1923-1945) : le Zuhûr al-basâtîn de Cheikh Moussa Kamara ", Islam et Sociétés au sud du Sahara, 7 : 95-110.

-, 1996 « La construction de l'altérité ethnique peule dans l'œuvre de Faidherbe », Cahiers d'Études africaines, XXXVI (3), 143 : 421-441.

-, 2002 « À la recherche d'Henri Gaden », Islam et Sociétés au sud du Sahara, 16 : 7-33.

-, 2004 Les représentations françaises sur les Peuls et les Haalpulaar'en ("Toucouleurs ») du XVIII au début du XX ${ }^{e}$ siècle : Des stéréotypes à la connaissance de l'Afrique, Thèse de doctorat, Paris, Université Paris VII.

PONDOPOULO-SANCHEZ, A., 2008 Français et Peuls à l'époque coloniale. Histoire d'une relation privilégiée. Les hommes, les stéréotypes, les savoirs à l'époque coloniale, Paris, Les Indes savantes (« Rivages des Xantons »).

POUILLON, F. (dir.), 2008 Dictionnaire des orientalistes de langue française, Paris, Karthala.

REBSTOCK, U., OSSWALD, R. \& WILD 'ABDALQADIR, A., 1988 Katalog der arabischen Handschriften in Mauretanien, Stuttgart, F. Steiner.

RICARD, A., 1995 Littératures d'Afrique noire, Paris, Karthala.

RICARD, A. \& JÉGO, A.-L., 2001 Henri Gaden, Photographe, Bordeaux, Confluences.

ROBINSON, D., 1988 « Un historien et anthropologue sénégalais : Shaykh Musa Kamara », Cahiers d'Études africaines, XXVIII (1), 109 : 89-116.

-, 2004 Sociétés musulmanes et pouvoir colonial français au Sénégal et en Mauritanie, 1880-1920: parcours d'accommodation, Paris, Karthala.

ROPER, G. J. (ed.), 1992-1994 World Survey of Islamic Manuscripts, 4 vol., London, Al-Furqan Islamic Heritage Foundation; Leiden, E. J. Brill.

SAINT-MARTIN, Y.-J., 1989 Le Sénégal sous le second Empire : naissance d'un empire colonial (1850-1871), Paris, Karthala.

SALVAING, B., 2002 «La question des manuscrits au Fuuta Jaloo (Guinée) », Communication pour le Chemin de l'Encre, Bamako, 8 août (inédit).

<http://www.sum.uio.no/research/mali/timbuktu/events/chemin/bernard.pdf>.

SALVAING, B. \& CABA BAH, I., 2004 « Les mystères des choses qui sont dedans les tombes... », Édition, traduction et commentaire d'un poème peul, Afrique \& Histoire, 2 (1) : 235-263.

SALVAING, B. \& AL-HADJI THIERNO MOUHAMmAdou, B., 2008 Une vie au Fouta Djalon, Brinon sur Sauldre, Grandvaux.

SALVAING, B. \& HUNWICK, J., 2003 « Writers of Guinea », in Arabic Literature of Africa, 4, The Writings of Western Sudanic Africa, Leiden, Brill : 491-529.

SAMB, A., 1970 « La vie d'El Hadj Omar », par Shaykh Muusa Kamara (éd. et trad. par Amar Samb), Bulletin de l'IFAN, B, 32 (1) : 44-135 ; 32 (2) : 370-411 ; 32 (3) : 770-818.

—, 1971 Essai sur la contribution du Sénégal à la littérature d'expression arabe, Dakar, IFAN, Mémoires de l'IFAN, 87. 
-, 1973 « L'islam et le christianisme » (éd. et trad. par A. Samb), Bulletin de l'IFAN, B, 35 (2) :

269-322.

-, 1976 « La condamnation de la guerre sainte » (éd. et trad. par A. Samb), Bulletin de l'IFAN, B, 38

(1) : 158-199.

SAVADOGO, B. [T], 1998 Confréries et pouvoirs. La Tijâniyya Hamawiyya en Afrique occidentale (Burkina Faso, Côte-d'Ivoire, Mali, Niger) : 1909-1965, Thèse de doctorat, 2 vol., Aix-en-Provence, Université de Provence.

SCHMITZ, J., 1998 « L'Afrique par défaut ou l'oubli de l'orientalisme », in J.-L. AMSELLE \& E. SIBEUD (dir.), op. cit. : 107-121.

schmitZ, J. (dir.) \& BousBinA, S. (trad.), 1998 Florilège au jardin de l'histoire des Noirs, Zuhûr al-Basâtîn, par Shaykh Muusa Kamara, L'aristocratie peule et la révolution des clercs musulmans (vallée du Sénégal), Paris, Éditions du CNRS.

SECK, A., 2008 La Question musulmane au Sénégal (entre fin des années 1980 et milieu des années 2000), Thèse de doctorat, Aix-en-Provence, Université de Provence.

SEYDOU, C., 1973 « Panorama de la littérature peule », Bulletin de l'IFAN, B, 35 (1) : 176-218.

-, 1977 Bibliographie générale du monde peul, Niamey, Institut de recherches en sciences humaines (« Études Nigériennes », 43).

SEZGIN, F., 1967 Geschichte des arabischen Schrifttums, 10 vol., Leiden, Brill.

SIBEUD, E., 2002 Une science impériale pour l'Afrique ? La construction des savoirs africanistes en France, 1878-1930, Paris, Éditions de l'EHESS.

SMITH, H. F. C., 1961 « A Neglected Theme of West African History : the Islamic Revolutions of the $19^{\text {th }}$ century ", Journal of the Historical Society of Nigeria, 2 (2) : 169-185.

SOH, Siré Abbâs, 1913 "Chroniques du Foûta sénégalais », Revue du Monde musulman, XXIV : 1-114 ; XXV : 165-235.

sow, A.-I. (dir.), 1966 La femme, la vache, la foi, Paris, Julliard.

-, 1968 Chroniques et récits du Fûta Djalon, Paris, Klineksieck.

-, 1971 Le Filon du bonheur éternel de Tierno Mouhammadou-Samba, Paris, A. Colin (rééd. Paris, Nubia, 1999).

STEWART, C. C., 1994 Catalogue of Arabic Manuscripts among the Ahl al-Shaykh Sidiyya, Boutlimit, Mauretania : Libraries of Harun b. Baba b. Sidi Muhammad b. Sidiyya al-Ntishaii, Ismail b. Baba, Yaqub b. Muhammad b. Baba Fihris al-makhtutat lada Ahl al-Shaykh Sidiya, Butilimit, Muritaniyah: Maktabat Harun ibn Baba ibn Sayyid Muhammad ibn Sidiya al-Intishai, Ismail ibn Baba, wa-Yaqub ibn Muhammad ibn Baba, Urbana (Ill.), University of Illinois at Urbana-Champaign.

STEWART, C. C., OUlD Sidi AHMAD, A. S. \& MUhAMmad YAHYA, O. A., 1990 « Catalogue of Arabic Manuscripts at the Institut Mauritanien de recherche scientifique », Islam et Sociétés au sud du Sahara, $4: 179-184$.

TAMARI, T., 1994 « Cinq textes bambara en caractères arabes : Présentation, traduction, analyse du système graphique », Islam et Sociétés au sud du Sahara, 8 : 97-121.

-, 2005 « La prose littéraire arabe en traduction bambara ; une maqâma d'al-Harîrî », in U. BAUMGARDT \& J. DERIVE (dir.), Paroles nomades. Écrits d'ethnolinguistique africains, Paris, Karthala : 431-463. 
TAPIERO, N., 1963 « Le grand cheikh peul Uthman ibn Fudi [...] et certaines sources de son islam doctrinal », Revue des Études islamiques, XXXI : 49-88.

TEMIMI, A., 1985 « L'ouvrage Nayl al-ibtihâj d'Ahmad Baba : une encyclopédie de biographies maghrébines ", Revue maghrébine de Documentation, 3 : 143-146.

TRIAUD, J.-L., 1987 « Les études sur l'islam en Afrique noire. Essai historiographique », Lettre d'information de l'Association française pour l'étude du monde musulman (AFEMAM), 2, déc. : 65-80.

-, 1995 La légende noire de la Sanûsiyya. Une confrérie musulmane saharienne sous le regard français (1840-1930), 2 vol., Paris, Aix-en-Provence, Éditions de la Maison des sciences de l'Homme.

-, 1997 « Le crépuscule des Affaires musulmanes en AOF, 1950-1956 », in D. ROBINSON \& J.-L. TRIAUD (dir.), Le Temps des marabouts. Itinéraires et stratégies islamiques en Afrique occidentale française, v. 1880-1960, Paris, Karthala : 493-519.

,$- 1998 \mathrm{a}$ " Le thème confrérique en Afrique de l'Ouest. Essai historique et bibliographique ", in A. POPOVIC \& G. VEINSTEIN (dir.), Les ordres mystiques dans l'islam, Paris, Éditions de l'EHESS : 271-282.

-, 1998b « Haut-Sénégal-Niger, un modèle “positiviste” ? De la coutume à l'histoire : Maurice Delafosse et l'invention de l'histoire africaine ", in J.-L. AMSELLE \& E. SIBEUD (dir.), op. cit. : 210-232.

-, 1998c « L'Islam vu par les historiens français », Esprit, $246: 110-132$.

-, 2000 « Islam in Africa under French Colonial Rule », in N. LEVTZION \& R. L. POuwels (eds.), The History of Islam in Africa, Athens, Ohio University Press : 169-187.

-, 2004 Compte rendu de l'ouvrage de P. F. de Moraes Farias, Arabic Medieval Inscriptions from the Republic of Mali. Epigraphy, Chronicles and Songhay-Tuareg History, in Annales, 59 (5-6) : 1206-1210.

-, 2005 « L'éveil à l'écriture : un nouveau Moyen Âge sahélien ». À propos de l'ouvrage de P. F. de Moraes Farias, op. cit., Afrique \& Histoire, $4: 195-243$.

TRIAUD, J.-L. \& D. ROBINSON (dir.), 2000 La Tijâniyya. Une confrérie musulmane à la conquête de l'Afrique, Paris, Karthala.

TRIMINGHAM, J. S., 1961 Islam in West Africa, Oxford, Clarendon Press.

VIEILlARD, G., 1939 Notes sur les coutumes des Peuls du Fouta Djallon, Paris, Larose, Publications du Comité d'études historiques et scientifiques de l'AOF, série A.

YATTARA, A. M. \& SALVAING, B., 2000 Almamy, une jeunesse sur les rives du fleuve Niger, Brinon-surSauldre, Grandvaux.

-, 2003 Almamy, l'âge d'homme d'un lettré malien, Brinon-sur-Sauldre, Grandvaux.

ZOUBER, M., 1977 Ahmad Bâbâ de Tombouctou (1556-1627). Sa vie et son œuvre, Paris, Maisonneuve et Larose.

\section{NOTES}

1. Signalons ici un essai de transversalité bien informé (KANE 2003).

2. Dès son arrivée, il fonde deux périodiques : l'Annuaire du Sénégal et dépendances (SaintLouis, 1858 à 1904) et le Moniteur du Sénégal et Dépendances. Ces journaux sont destinés à la publication des actes officiels et à l'information sur la vie de la colonie. Ils contiennent aussi des contributions d'ordre géographique et ethnographique. 
3. Sur l'importance durable de ces classifications et représentations, voir les commentaires de Jean-Loup AMSELLE (1996).

4. Joël GLASMAN (2002: n. 390) cite plusieurs de ces travaux inédits, qui se trouvent notamment aux Archives nationales du Sénégal et aux Archives nationales d'OutreMer.

5. Sur la recherche d'une Afrique " authentique » et le rejet de tout apport religieux extérieur, comme composantes de la politique coloniale française anti-islamique, voir les remarques de Jean-Loup AMSELLE (1990: 181-204). Ou encore : «Il faut mesurer ce que l'anthropologie africaniste a perdu avec la mise aux oubliettes et la disqualification de Delafosse par Griaule et ses disciples. Il faut mentionner tout d'abord la dépolitisation, la déshistoricisation et la désislamisation des sociétés de l'Afrique de l'ouest subsaharienne » (ibid. 1998 : 13).

6. Sur cette marginalisation de l'islam par les ethnologues, et cette recherche d'un " huis-clos " non islamisé dans les sociétés africaines, voir les observations de JeanLoup AMSELLE (1996: 38).

7. À la fin de l'année 1905, le gouverneur général Roume désigna Robert Arnaud comme le responsable des Affaires musulmanes au sein du Bureau politique du Gouvernement général (ROBINSON $2004: 157)$. C'était le premier titulaire d'une fonction spécialisée en ce domaine. Un Service des Affaires musulmanes distinct et organisé n'apparaît qu'en 1912, auprès de la Direction des Affaires politiques. Son premier titulaire fut Paul Marty (ibid.).

8. Il y avait aussi, en métropole, une vision policière du mouvement ouvrier, à cette différence près que l'histoire du mouvement ouvrier ne s'est pas construite sur de telles bases.

9. Le CHEAM (Centre des hautes études d'administration musulmane) a été créé, en 1936, par le gouvernement du Front populaire. Son premier directeur, de 1936 à 1940, puis, après l'Occupation, jusqu'en 1953, fut Robert Montagne, spécialiste reconnu du Maroc. Il fut suivi, de 1954 à 1967, par le général Pierre Rondot, spécialiste du renseignement. Il s'agissait, pour le gouvernement français, de se doter d'un organe de centralisation et de formation en matière d'Affaires musulmanes couvrant les différentes possessions françaises concernées. Rebaptisé, après les décolonisations, Centre des hautes études sur l'Afrique et l'Asie modernes, ce service a été dissous en 2000 et ses archives versées aux Archives nationales de France (site de Fontainebleau).

10. Vincent Monteil (1913-2005) Saint-cyrien, affecté en 1938 dans une unité méhariste au Maroc. Il participe aux combats pour la libération de la France (blessé en 1945). Observateur militaire auprès des Nations Unies à Jérusalem en 1948. Carrière dans les ambassades (1950). Engagé dans le bataillon français de Corée (1953), puis en Indochine. Ce qu'il y observe fait de lui un anti-colonialiste convaincu. Membre de cabinets ministériels ou simple écrivain, il combat les méthodes de la guerre menée en Algérie. Il est à Rocher Noir auprès de Christian Fouchet en 1962 pour assurer la passation des pouvoirs à l'Algérie indépendante. En 1958, il succède à Jacques Berque à la direction du Centre français d'étude de l'arabe à Bikfaya (Liban). En 1963, il est directeur de l'Institut français (puis fondamental) d'Afrique noire (IFAN), à Dakar, et le restera jusqu'en 1968. Il finit sa carrière académique à l'Université Paris VIII-Vincennes. Voir la notice biographique que nous avons publiée dans François Pouillon (2008). 
11. Entre 1961 et 1968, V. MONTEIL publie dix articles dans le Bulletin de l'IFAN, notamment sur l'histoire de l'islam au Sénégal, sur les mourides et sur les manuscrits arabo-africains, trois autres aux Archives de Sociologie des Religions sur les mêmes thèmes (1962, 1963, 1965). Sous le titre Esquisses sénégalaises, l'IFAN publie, en 1967, un recueil de plusieurs de ces articles.

12. Sur Maurice Delafosse, voir la biographie par sa fille, Louise DELAFOSSE (1976) et l'ouvrage collectif dirigé par Jean-Loup AMSELLE et Emmanuelle SIBEUD (1998).

13. Nous détenons une copie des correspondances échangées entre Maurice Delafosse et Charles Monteil grâce à l'obligeance de Vincent Monteil.

14. Auguste Terrier (1873-1932) est secrétaire général du Comité de l'Afrique française.

15. Il s'agit d'un texte important: Chroniques du Foûta sénégalais, traduction et commentaires du manuscrit de Siré Abbas Soh par M. DELAFosse et H. GADEN, Paris, Ernest Leroux (Collection de la Revue du Monde Musulman), 1913, XXIV, pp. 1-114, et XXV, pp. 165-235.

16. Sancergues (Cher), Maurice Delafosse à Charles Monteil, 27 juillet 1912. Louise DELAFOSSE (1976 : 293), à qui j'avais communiqué cette correspondance (et qui, de son côté, m'avait donné accès à différents documents privés) ne donne pas les références précises ni le statut de cet extrait dans son ouvrage. Voilà qui est fait.

17. Delafosse à Gaden, 16 décembre 1910, dans PONDOPOUlo (2004). Un seul mot sur six ou sept est déjà un bon score, mais ce n'est là qu'une illustration de ses difficultés.

18. «Ici, dès mon retour à Paris, je montrerai le précieux manuscrit à mon beau-père, mieux placé que quiconque pour en apprécier la valeur et reconnaître s'il mérite d'être publié [...]. » Delafosse à Gaden, 15 août 1910, dans PONDOPOUlo (2004 : 253).

19. Voir notamment le tableau chronologique suggestif « Africanisme et orientalisme à l'aube du XX $X^{e}$ siècle ", établi par J. Schmitz, P. Legrosse et O. Kyburz ( schmITz dans SCHMITZ \& BOUSBINA 1998 : 40-41). Voir aussi Bernard MOURALIS (2000b : 17-28).

20. Lui-même auteur d'un Précis de grammaire arabe (1897), qui fera autorité pour plusieurs générations d'étudiants.

21. Voir ses manuels de langue agni (DELAFOSSE 1900), mandingue (ibid. 1901a) et hausa (ibid. 1901b).

22. La direction des Bureaux arabes a été créée en 1837.

23. C'est ce que reconnaît J. FRÉMEAUX (1998 : 202-203) lorsqu'il écrit, nuançant ainsi son hypothèse initiale: "Sans même parler de filiations, il y a, par ailleurs, beaucoup d'analogies » entre la situation d'un administrateur civil en Afrique noire et celle « d'un chef de bureau arabe des débuts de l'occupation ». Nous retenons volontiers ce terme d'analogie.

24. Al-Bartili (m. 1805), Fath al-shakûr, voir EL HAMEL $(1994,2002)$.

25. Muusa Kamara, Zuhûr al-Basâtîn, voir David ROBINSON (1988), Jean SCHMITZ (dir.) (1998), et le dossier « À la découverte de Shaykh Musa Kamara » (BOUSBINA, SCHMITZ \& PONDOPOULO 1993). Voir aussi Constance HILLIARD (1987 : 175-186).

26. Voir la préface de l'ouvrage pour les précisions sur les conditions de recueil du manuscrit. 
27. Clozel fut successivement gouverneur par intérim, puis titulaire de la Côte-d'Ivoire (1902-1905), gouverneur du Soudan français (1908-1912), gouverneur général de l'AOF, par intérim, puis titulaire (1912-1917). Sur Clozel, voir Alice conKLIN (1997 : 176-178).

28. C'est Clozel qui lança la collection où allait prendre place Haut-Sénégal-Niger et en rédigea la préface.

29. Ce comité publie d'abord un Annuaire et Mémoires (annuel, 1916 et 1917), qui devient, à partir de 1918, le Bulletin du Comité d'Études historiques et scientifiques de l'AOF, trimestriel.

30. Parmi les travaux les plus importants, signalons, les "Cahiers de Yoro Dyao" (1912), les «Chroniques du Foûta sénégalais », avec Delafosse, déjà citées (1913), le Dictionnaire peul (1913-1914), les Proverbes et maximes peuls (1931) et La vie d'El Hadj Omar. Qacida en poular de Mohammadou Aliou Tyam (1935). Ce dernier ouvrage est une édition critique de la qasida (poème) conforme aux normes orientalistes, avec texte pulaar transcrit (en caractères latins), traduction française juxtaposée et notes explicatives. Sur Gaden, outre PONDOPOULO (2002), on consultera le beau livre d'Alain RICARD et AnneLaure JÉGo (2001), qui est l'ouvrage de référence de l'exposition « 30 Photographies de Henri Gaden » présentée par les auteurs (Bordeaux, Salon du livre, 2001 et Tours, Université François Rabelais, 2006). Le choix a été fait à partir de 335 clichés disponibles.

31. On lui doit la traduction partielle de l'œuvre de géographie politique d'un secrétaire d'État égyptien du XIV siècle, Al-'Umari. Il s'agit des parties concernant plusieurs régions du continent africain : l'Éthiopie chrétienne et musulmane, le Bornou, l'empire du Mali, le Maroc (Mérinides et Almohades), le royaume abdelwadide de Tlemcen et l'État hafside de Tunis. C'est une des grandes sources arabes classiques de l'histoire du Mali médiéval qui s'ajoute donc aux Ta'rikh-s (AL-OMARI 1927).

32. Vincent MONTEIL (1971: 87) a fait des réserves sur cette œuvre, sans que l'on comprenne bien son argument. Il cite trois fois Kamara, brièvement, et ajoute : « La Chronique du Foûta-Tôro (Zuhûr al-Basâtîn) du Cheikh Moussa Kamara de Ganguel (1921) est encore inédite : il n'est pas sûr qu'elle représente une source indépendante.» Moussa Kamara a certes compilé, mais c'est cette compilation même qui est précieuse.

33. Voir aussi A. RICARD, qui inclut cette attitude dans un mouvement plus général, qui s'opère alors, de l'intérêt scientifique "des écrits arabes à la graphie arabe ", donc à l'ajamî (1995: 75-77 pour le peul, 78-83 pour le hausa).

34. Massignon a plus fréquenté le Maroc (où il fait son premier voyage d'études en 1904) et le Proche-Orient que l'Algérie. Ce n'est donc pas un représentant de l'« École d'Alger ».

35. Ismaël HAMET (1906) est un partisan de la diffusion de l'enseignement français et de l'assimilation.

36. Hamet a recueilli dans cet ouvrage, comme le feront ensuite René Basset et Paul Marty, la tradition maure relative à Nasr al-Dîn et à la guerre de Shur Bubba.

37. On doit à René BASSET (1883) un premier catalogue des manuscrits des bibliothèques de Fès.

38. Ikhbâr al-ahbâr bi Ikhbâr al-abâr (« Le livre des lettrés renseignés sur l'histoire des puits »). L'Institut des Études africaines de Rabat a publié une nouvelle version de cet 
ouvrage : Ahmed Oueld al-Hassan, (Casablanca, 1991, 124 p.). Celui-ci a révisé le texte arabe et corrigé la traduction de Paul Marty.

39. Nous remercions Constant Hamès d'avoir attiré notre attention sur Hamet et Basset, à ses yeux trop ignorés.

40. L'un des maîtres ouvrages de la science coloniale, celui de DEPONT et COPPOLANI (1897), s'intitule Les confréries religieuses musulmanes.

41. Sur cette « peur française de l'islam », voir notre article dans Esprit (TRIAUD 1998c).

42. Sur le retournement des positions de Le Chatelier, voir nos commentaires dans TRIAUD $(1987: 66,1998 \mathrm{a}: 174)$.

43. Sur la biographie d'Alfred Le Chatelier, voir Jean LE CHATELIER (1987).

44. La Mission scientifique du Maroc, implantée à Tanger en 1904, a été fondée par Le Chatelier, d'abord à ses frais, puis avec une subvention du ministère de l'Instruction publique. La Mission produisait, à son origine, une seule publication: Les Archives marocaines. En 1906, Le Chatelier y ajouta la Revue du Monde musulman.

45. Delafosse à Gaden (début 1910). De fait, DELAfosse (1910a, b, c) publie quatre articles dans la Revue du Monde musulman, en 1910.

46. Auguste Terrier (1873-1932), secrétaire général du Comité de l'Afrique française, directeur de l'office chérifien du Maroc, censeur du Crédit foncier d'Algérie et de Tunisie.

47. Sur la question de l'islam noir, voir TRIAUD (1987 : 67-68).

48. Nous renvoyons le lecteur aux matériaux originaux et à la démonstration novatrice d'Anna Pondopoulo (2004: 262). Elle montre encore comment Delafosse trouvait aussi une inspiration, sur les questions de confréries et de marabouts, dans un ouvrage de politique musulmane de son beau-père, Octave HOUDAs (1904), L'Islamisme.

49. Il est difficile de donner une date de naissance précise à la confrérie. Faut-il retenir celle de la révélation mystique reçue par Amadou Bamba (vers 1891), la longue période de ses tribulations successives (1895-1912), ou la dernière période de sa vie à Diourbel dans une liberté à peu près retrouvée (1912-1927) ?

50. On notera l'intérêt d'un arabisant de Langues O' pour Usman dan Fodio : Norbert TAPIERO (1963). V. MONTEIL (1971: 92 n.) signale que N. Tapiero préparait alors « une thèse sur l'esprit et la doctrine d'Osman dans Fodio, d'après les neuf manuscrits de la Bibliothèque nationale ", mais cette thèse ne vit pas le jour.

51. On peut ranger dans un registre parallèle l'ouvrage de l'arabisant Fernand DUMONT (1975), ancien officier d'Affaires musulmanes, docteur en sciences islamiques, qui est alors conseiller à la présidence de la République du Sénégal. Il offre une analyse et des traductions de nombreux vers inédits d'Amadou Bamba (ibid.), même s'il ne s'agit pas d'une édition critique avec reproduction des originaux.

52. On rappellera ici l'article de Christiane SEYDOU (1973) ainsi que sa bibliographie générale du monde peul (1977).

On attend avec intérêt une publication de manuscrits guinéens sous la direction de Bernard Salvaing (SALVAING \& HUNWICK 2003). Voir aussi B. SALVAING (2002). B. Salvaing a plus particulièrement mis l'accent, au cours de ces dernières années, sur la biographie d'hommes-ressources au Mali et en Guinée. Voir A. M. YATTARA et B. SALVAing (2000, 2003), et B. SALVAing \& A.-H. T. MOUHAMmadou (2008). 
53. Les notes, souvent reprises de commentaires antérieurs, ont beaucoup vieilli avec le temps. Une autre édition du même corpus, à quelques différences près, a été faite en anglais (LEVTZION \& HOPKINS 1981). Ces notes ont été actualisées.

54. Voir l'introduction (pp. VII-IX) sur les conditions de réalisation de ce projet.

55. Sur l'itinéraire intellectuel de Moussa Kamara, voir l'introduction de Jean schMITZ (1998: 9-79).

56. Delafosse avait déjà fait cette comparaison (Schmitz, dans SCHMITZ \& BOUSBINA 1998 : 44).

57. Cet article comporte la liste des écrits de Kamara, ainsi qu'une table des matières du tome 1 du Zuhûr. Ces informations sont reprises et revues dans SCHMITZ (ibid. : 80-83).

58. On pense, par analogie, à une autre somme, produite à l'autre extrémité du désert saharien occidental. Mohamed al-Mokhtar Soussi (1900-1963), un maître soufi et érudit $\mathrm{du}$ Sud marocain, auteur d'une œuvre prolifique, dont al-Ma'sul, ouvrage encyclopédique en vingt volumes, "mine " pour de nombreux chercheurs. Le point commun avec Moussa Kamara, au-delà des différences de contexte et de génération, c'est la manière dont un érudit "traditionnel » est saisi par la passion de l'écriture encyclopédique sur sa région, dans une situation coloniale de contact avec la culture occidentale.

59. Les arabisants traducteurs: Said Bousbina, à Paris, et Khadim Mbacké et Abdoul Malal Diop à Dakar. Les spécialistes du pulaar : Olivier Kyburz et Ibrahima Abou Sall, à Paris. Les islamisants et historiens : Charles Becker (historien et démographe), à Dakar, Dedoud Ould Abdallah (historien) à Paris et Nouakchott, David Robinson (historien du Futa Toro), aux États-Unis, Constant Hamès (islamisant) à Paris, Oumar Kane (historien du Futa Toro) à Dakar. Les anthropologues : Jean Schmitz et Olivier Leservoisier, à Paris (sCHмITZ 1998 : 71-74).

60. Folios 167-330 (schMITZ 1998 : 75).

61. Là, d'ailleurs, ne s'épuise pas la production de Moussa Kamara, « auteur d'écrits en arabe et en pulaar sur des domaines et des zones géographiques très variés " (ROBINSON 1988: 109-110 ; schMITZ dans schMITZ \& BOUSBINA 1998: 80-83) : le Zuhûr albasâtîn ne constitue en fait qu'un seul de ses ouvrages.

62. Ousmane Kane voit deux raisons principales dans cette occultation : la tradition des Lumières, qui vise à une étude rationnelle de l'islam, et l'opposition "orthodoxe ", de Ibn Taimiyya jusqu'aux wahhabites, qui dénonce ce genre de pratiques (KANE 2003 : 29-30).

63. Chaque volume de la série de Fuat Sezgin correspond à une discipline scientifique (t. 1: sciences islamiques et histoire, 1967; t. 2: poésie, 1975; t. 3: médecine, pharmacie, zoologie, art vétérinaire, 1970 ; t. 4 : alchimie, chimie, botanique, agriculture, $1971 ;$ t. 5 : mathématiques, $1974 ;[. .$.$] t. 8$ : lexicographie, 1982; t.9: grammaire, 1984 - avec des réimpressions). Chaque volume comprend deux parties : la première est une histoire de la discipline considérée, la seconde une reconstitution biobibliographique à propos de chaque savant. Textes en allemand.

64. Compiled by John Hunwick, with the assistance of Ousmane Kane, Bernard Salvaing, Rüdiger Seesemann, Mark Sey et Ivor Wilks.

65. Voir le texte du projet en français (site de l'Université d'Oslo) :

$<$ http://www.sum.uio.no/research/mali/timbuktu/project/timanus.fr.pdf>. 
66. Les auteurs sont en majorité sahariens et subsahariens. La majorité des titres concernent les sciences islamiques (figh et soufisme), voir le site de l'Université d'Oslo : <http://www.sum.uio.no/research/mali/timbuktu/events/rabat/ bibliographie.html>.

67. Catalogue en 4 volumes contenant 4000 manuscrits concernant les sciences islamiques (Coran, hadith, figh, littérature, astrologie, grammaire, soufisme), des fatwas et des correspondances (source : Université d'Oslo, ibid.).

68. C'est également vrai dans d'autres domaines. On pense à l'archéologie-préhistoire des bordures sahariennes, enjeu, dans les années 1950 et 1960, entre les spécialistes venus d'Algérie (Henri Lhote) et ceux venus de l'IFAN (Raymond Mauny).

\section{RÉSUMÉS}

Résumé

L'Afrique occidentale française a connu une « saison orientaliste », qui couvre une période allant des années 1890 aux années 1920. L'hypothèse a été émise d'une influence déterminante des modèles de l'«École d'Alger» et des Bureaux arabes. L'article relativise cette hypothèse «diffusionniste » et montre plutôt comment, passé le moment des premiers influx venus du Maghreb, un orientalisme proprement "sénégalo-soudanais » se met en place à l'initiative de trois hommes, Houdas, Delafosse et Gaden, qu'une commune passion pour l'érudition textuelle et les dictionnaires et un intérêt personnel pour les cultures africaines réunissent. Cette "saison orientaliste" fut d'abord l'entreprise volontaire de ces trois acteurs coloniaux motivés, une conjonction singulière qui n'a pas eu de continuateurs immédiats. Plus encore que les institutions algériennes, c'est l'École des Langues orientales de Paris qui apparaît comme une matrice commune.

Ce groupe savant considère alors le champ subsaharien, non comme une périphérie, mais comme une province culturelle particulière avec sa propre centralité. «Les populations, auxquelles on est tenté de refuser toute initiative en matière de progrès, ont une civilisation propre qui ne leur avait pas été imposée par un peuple d'une autre race [...]» (Houdas 1898). La théorie de l'« islam noir ", au début du xx siècle, est une formulation allant dans le même sens, même si ce groupe n'en est pas l'auteur direct. Cette théorie, qui vise à couper l'islam subsaharien des tuteurs arabes, vise tout autant à autonomiser l'étude de cet islam par rapport au monopole alors exercé par les tuteurs orientalistes du Maghreb. Les tenants de cette revendication d'autonomie scientifique cherchent à promouvoir une revalorisation culturelle des sociétés subsahariennes, une attention aux textes locaux comme instruments d'habilitation d'une histoire et d'une ethnographie de ces sociétés, et l'adoption de concepts adaptés à ce terrain. Cependant, ce décentrement du savoir islamologique accumulé au Maghreb ne va pas de soi, aussi bien pour les arabisants du Maghreb que pour les ethnologues de l'Afrique subsaharienne. L'action de ce groupe savant se distingue, en outre, de l'expertise proprement coloniale d'«Affaires musulmanes ", incarnée, entre autres, par Paul Marty. Une telle expertise, qui tient parfois lieu de bibliothèque de référence, à défaut d'une bibliothèque orientaliste diversifiée, a aussi servi de repoussoir, en rendant l'objet « islam » suspect - aussi bien aux yeux des chercheurs de l'époque qu'à ceux de la période des indépendances. Cette littérature de surveillance a contribué à entretenir l'idée que la «question islamique » relevait d'autres paradigmes que ceux des « Études africaines ». 
La fin de l'article met en perspective la chaîne des héritiers, du côté francophone, notamment, en un premier temps, dans le domaine des études peules, et, du côté anglophone, par la relève qui s'opère dans le catalogage extensif de manuscrits subsahariens.

Cette reconnaissance des chaînes de transmission jusqu'à l'époque actuelle, ainsi que le nouveau transfert qui s'opère du côté africain (Hampâté Bâ), témoigne des liens et des ruptures qui existent entre cette première "saison orientaliste" et celle qui se développe, de façon multiforme et plus éclatée, à partir des années 1960.

\section{Abstract}

French West Africa had an "Orientalist season" in the period running from the 1890s to the 1920s. A hypothesis was raised about the determining influence of the "Algiers School" and the Arab Bureaux. This article places this "diffusionist" hypothesis into perspective and shows instead that, after the first North African influx a true Senegal-Sudan Orientalism was established at the initiative of three men, Houdas, Delafosse and Gaden, who shared a passion for textual erudition and dictionaries, as well as a personal interest in African cultures. At the outset this "Orientalist season" was a voluntary undertaking by these three motivated colonial actors, a remarkable union that lacked immediate successors. Their common matrix was the School of Oriental Languages in Paris, more than the Algerian institutions.

This group of scholars viewed the sub-Saharan region not as a periphery but a specific cultural province with its own centrality. "Those populations to whom we have tended to deny any initiative in progress, have their own civilisations that were not imposed by them by a people from another race [...]" (Houdas 1898). The "Black Islam" theory in the beginning of the $20^{\text {th }}$ century was a step in the same direction, even though this group did not instigate it directly. This theory, which aimed to cut sub-Saharan Africa off from its Arabic tutors, also tried to free the study of that Islam from the monopoly of the North African Orientalists. Proponents of this demand for scientific independence sought to promote a cultural re-evaluation of sub-Saharan societies, a specific attention to local texts as instruments for the accreditation of the history and ethnography of those societies, and to adoption of concepts adapted to the field. However, it was no easy matter for the North African scholars, any more than for the ethnologists of sub-Saharan Africa, to detach Islamic scholarship from its North African centre. The action of this group of scholars stands out from the colonial expertise in "Muslim Affairs" as incarnated by Paul Marty among others. That expertise served as a reference library, in the absence of a diversified Orientalist library, but also as a foil by making "Islam" a suspect subject for researchers at the time as well as during the independence period. This literature de surveillance helped to perpetuate the idea that the "Islamic question" belonged to other paradigms than "African Studies".

The end of the article is devoted to placing the chain of heirs into perspective, notably francophone ones in the domain of Fula studies to start with, and then Anglophone research, which has followed on with an extensive catalogue of sub-Saharan manuscripts.

By identifying the chains of transmission up to the present day, as well as the new transfer occurring on the African side (Hampâté Bâ), we show the connections and rifts that existed between that first "Orientalist season" and the one that developed in a multiform and more fragmented way after the 1960s. 
INDEX

Keywords : Maurice Delafosse, Henri Gaden, Octave Houdas, École Des Langues Orientales, Islam, Islamology, Scrolls, Orientalism

Mots-clés : Maurice Delafosse, Henri Gaden, Octave Houdas, École des Langues orientales, islam, islamologie, manuscrits, orientalisme

\section{AUTEUR}

JEAN-LOUIS TRIAUD

Centre d'étude des Mondes africains (CEMAF), Université de Provence. 